\title{
Numerical Simulation of Crystal Nucleation in Colloids
}

\author{
Stefan Auer ${ }^{1}$ and Daan Frenkel ${ }^{2}$ \\ 1 Department of Chemistry, Cambridge University, Lensfield Road, Cambridge, CB2 1EW, \\ United Kingdom,sa372@cam.ac.uk \\ 2 FOM Institute for Atomic and Molecular Physics, Kruislaan 407, 1098 SJ Amsterdam, \\ The Netherlands, frenkel@amolf.nl
}

\begin{abstract}
This article reviews the recent progress that has been made in the application of computer simulations to study crystal nucleation in colloidal systems. We discuss the concept and the numerical methods that allow for a quantitative prediction of crystal nucleation rates. The computed nucleation rates are predicted from first principles and can be directly compared to experiments. These techniques have been applied to study crystal nucleation in hard-sphere colloids, polydisperse hard-sphere colloids, weakly charged or slightly soft colloids and hardsphere colloids that are confined between two plane hard walls.
\end{abstract}

Keywords Crystal nucleation, Colloids, Monte Carlo simulation, Nucleation barrier, Kinetic prefactor, Crystal nucleation rate

$1 \quad$ Introduction . . . . . . . . . . . . . . . . . . . . . . . . . . . . . . 149

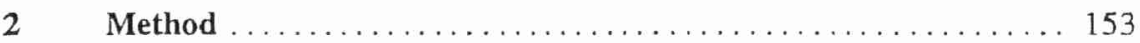

2.1 Calculation of the Cluster Size Distribution . . . . . . . . . . . . . . . 154

$2.2 \quad$ Kinetic Prefactor ............................... 161

3 Hard-Sphere Colloids ......................... 163

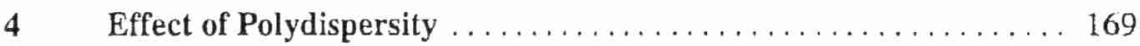

$5 \quad$ Weakly Charged Colloids . . . . . . . . . . . . . . . . . . . . . . . . 175

6 Poly-12-hydroxystearic Coated Polymethylmethacrylate Particles . 186

$7 \quad$ Wall Induced Crystallization . . . . . . . . . . . . . . . . . . . . . . . . . . . 190

$8 \quad$ Concluding Remarks . . . . . . . . . . . . . . . . . . . . . . . . . . 197

A Distribution of Cluster Sizes in Equilibrium . . . . . . . . . . . . 198

B Calculation of the Chemical Potential .................. 201

C Surface Free Energies of Critical Nuclei ... . . . . . . . . . . . . 202

References..................................... 205 
1

\section{Introduction}

Heating a block of ice will result in melting. Cooling the resulting water will freeze it again. At a given pressure, water and ice can coexist at only one temperature. The water-ice coexistence temperature at ambient pressure is of such importance for every-day life, that it has been chosen as the zero-point of the widely used temperature scale invented by the Swedish physicist Celsius. Closer inspection of the melting and freezing transition shows that this transition is not quite symmetric. Ice heated above $0^{\circ} \mathrm{C}$ always melts, whereas cooling it below $0^{\circ} \mathrm{C}$ does not always result in immediate freezing. In fact water, and most other liquids, can be cooled significantly below their freezing temperature and kept there without crystallizing $[1,2]$. This phenomena is known as supercooling. A supercooled liquid can be triggered into freezing by adding a little bit of the corresponding solid. A single snowflake in a glass of supercooled water will induce freezing of water that touches it and grow rapidly into a big chunk of ice. Other disturbances, such as dust or even shocks, can trigger the freezing of supercooled liquids as well. It thus seems that the freezing process has great difficulty to start spontaneously, but becomes very easy once it is initiated. The spontaneous formation of a piece of solid is an example of nucleation.

The fact that a liquid can be supercooled is best understood qualitatively in the framework of classical nucleation theory (CNT) (see e.g. Ref. [3]). According to CNT the free energy of a spherical nucleus that forms in a supersaturated solution contains two terms. The first term accounts for the fact that the solid phase is more stable than the liquid. This term is negative and proportional to the volume of the nucleus. The second term is a surface term. It describes the free energy needed to create a solid/liquid interface. This term is positive and proportional to the surface area of the nucleus. The (Gibbs) free energy of a spherical nucleus of radius $R$ has the following form:

$$
\Delta G=\frac{4}{3} \pi R^{3} \rho_{s} \Delta \mu+4 \pi R^{2} \gamma,
$$

where $\rho_{s}$ is the number density of the bulk solid, $\Delta \mu$ the difference in chemical potential between the solid and the liquid, and $\gamma$ is the solid/liquid surface free energy density. The function $\Delta G$ has a maximum at $R=2 \gamma /\left(\rho_{s}|\Delta \mu|\right)$ and the corresponding height of the nucleation barrier is given by

$$
\Delta G^{*}=\frac{16 \pi}{3} \frac{\gamma^{3}}{\left(\rho_{s}|\Delta \mu|\right)^{2}} .
$$

For small nuclei the surface term dominates and the free energy increases. Only if this nucleus exceeds a critical size does its free energy decrease and the crystallite can grow spontaneously (see Fig. 1). The probability for the formation of a critical nucleus depends exponentially on its free energy of formation:

$$
P_{c} \propto \exp \left(-\Delta G^{*} / k_{B} T\right)
$$




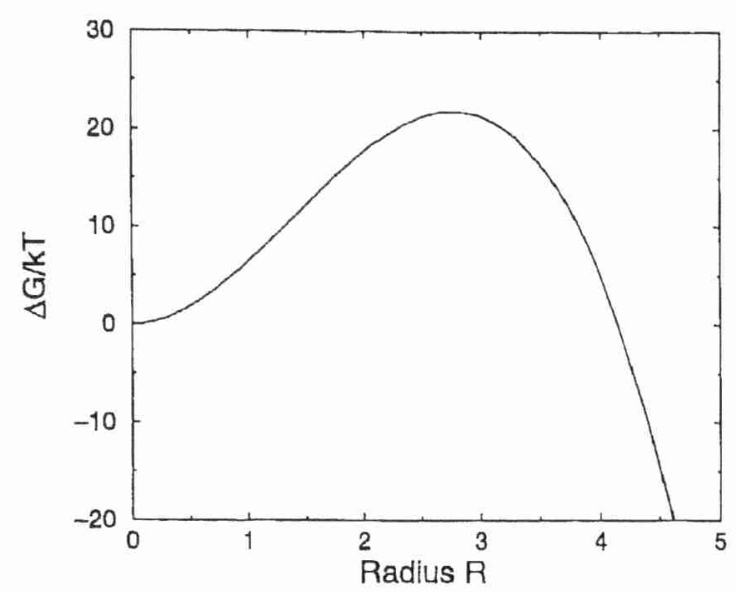

Fig. 1. Free energy barrier of a spherical nucleus described by classical nucleation theory Eq. (1). For small radii the surface term dominates and the free energy increases. When the radius exceeds a critical size the bulk term dominates and the free energy decreases

The crystal nucleation rate is given by the product of $P_{c}$ and a kinetic factor $\Gamma$ that describes the rate with which a critical nucleus grows. The CNT expression for the nucleation rate per unit volume is:

$$
I=\Gamma \exp \left[-\frac{16 \pi}{3 k_{B} T} \frac{\gamma^{3}}{\left(\rho_{s}|\Delta \mu|\right)^{2}}\right]
$$

with $\Gamma=Z \rho_{l} f_{c}^{+}$. Here $\rho_{l}$ is the number density of the liquid, $Z=\sqrt{|\Delta \mu| / 6 \pi k_{B} T n_{c}}$ is the Zeldovich factor and $f_{c}^{+}$is the attachment rate of particles to the critical cluster. The Zeldovich factor arises from the fact that not all particles that are at the top of the nucleation barrier crystallize: some will recross the barrier and melt again. The attachment rate of particles to the critical nucleus can be estimated by multiplying the number of monomers available at the surface of the nucleus - which is proportional to $n_{c}{ }^{2 / 3}$ - with a typical transition rate of these particles to become part of the nucleus. This transition rate is proportional to $D_{S} / \lambda^{2}$, where $D_{S}$ is a self diffusion coefficient and $\lambda$ is a typical distance over which diffusion takes place:

$$
f_{c}^{+}=\frac{24 D_{S} n_{c}^{2 / 3}}{\lambda^{2}} \text {. }
$$

The above expression for the nucleation rate is the one most commonly used to analyze crystal nucleation rate experiments. The problem with the CNT approach is however that, in most cases, neither $\lambda$ nor $\gamma$ are accurately known. More often than not, both parameters are obtained by fitting the $\mathrm{CNT}$ expression to experimental nucleation data.

To illustrate the problems associated with the fitting of CNT to experimental data, we give two examples. Let us start with Turnbull's first quantitative measurement of 
a nucleation rate in liquid mercury [2] (see Fig. 2). For the interpretation of his data he used Eq. (4), where he estimated the difference in chemical potential between the two phases by $\Delta \mu \approx \Delta h\left(T_{m}-T\right) / T_{m}$. Here $\Delta h$ is the enthalpy change per particle on freezing at coexistence, $T_{m}$ is the coexistence temperature and $T$ is the temperature of the liquid mercury. A plot of $\log (I)$ vs. $1 / T \Delta T^{2}$ should give a straight line with the slope proportional to $\gamma^{3}$ and the intercept equal to $\log (\kappa)$. From this two

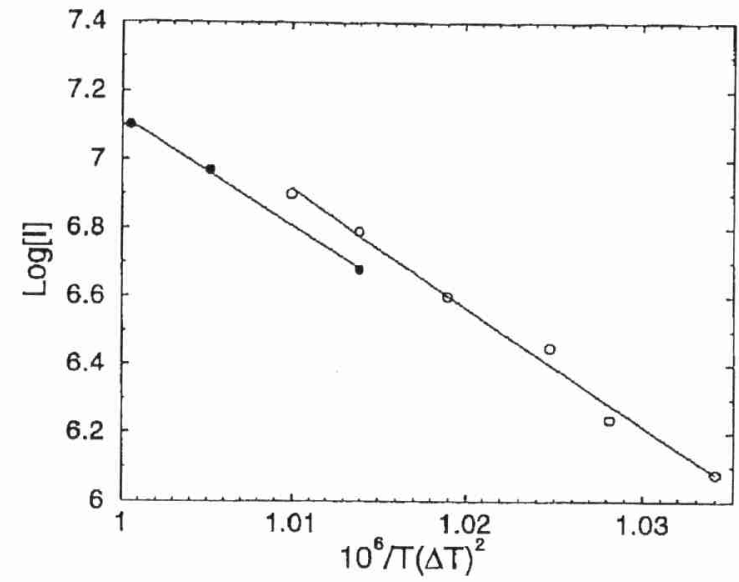

Fig. 2. The steady state nucleation rate, $I$ in units of $1 /\left(\mathrm{m}^{3} \mathrm{~s}\right)$, as a function of undercooling in Kelvin for liquid mercury from Ref. [2]. The open and the filled circles correspond to two different samples. The solid lines result from a two parameter fit of Eq. (4) to the experimental data

parameter fit we see that the functional form given by CNT for the nucleation rate reproduces the experimental data. However, the resulting value of $\kappa$ is a factor $10^{7}$ larger than predicted from CNT. The corresponding estimate for the typical diffusion distance $\lambda$ is many orders of magnitude too small. To explain this Turnbull noted is his paper: "...suppose that $\gamma$ depends upon temperature according to the equation: $\gamma=\gamma_{0}+b T$, [where $\gamma_{0}$ is the value at coexistence and $\mathrm{b}$ is a constant], ... a value of $b=0.0008 / K$ is sufficient to change the apparent value of the kinetic factor by six orders of magnitude." A remarkable statement which might be correct, but at the time direct corroboration was not possible because of the absence of a priori knowledge of both fit parameters. The major problem of experimental investigations of crystallization kinetics in atomic systems is the high speed of nucleus formation and subsequent crystal growth, as well as the difficulty of preventing heterogeneous nucleation. The second example we take from more recent experiments on the crystallization kinetics in a suspension of hard-sphere colloids. Crystallization in colloidal suspensions is interesting because it can be studied in considerable detail, since colloidal particles are much larger than atoms. Colloids therefore crystallize on a timescale which is about ten orders of magnitude longer than that for an atomic liquid. Moreover because of 
their size, colloids can be probed by powerful optical methods such as time-resolved static laser light scattering and confocal microscopy. In these systems it is also somewhat easier to control heterogeneous nucleation. In Fig. 3 we show the results from crystallization rate measurements in hard-sphere colloids, performed by two different groups $[5,6]$. For this system the difference in chemical potential between the two phases can be calculated accurately from existing analytical expressions for the equation of state. The curves in the figure result from a two parameter fit of Eq. (4) to the experimental data. Palberg [4] fitted the data from Harland and van Megen [5]

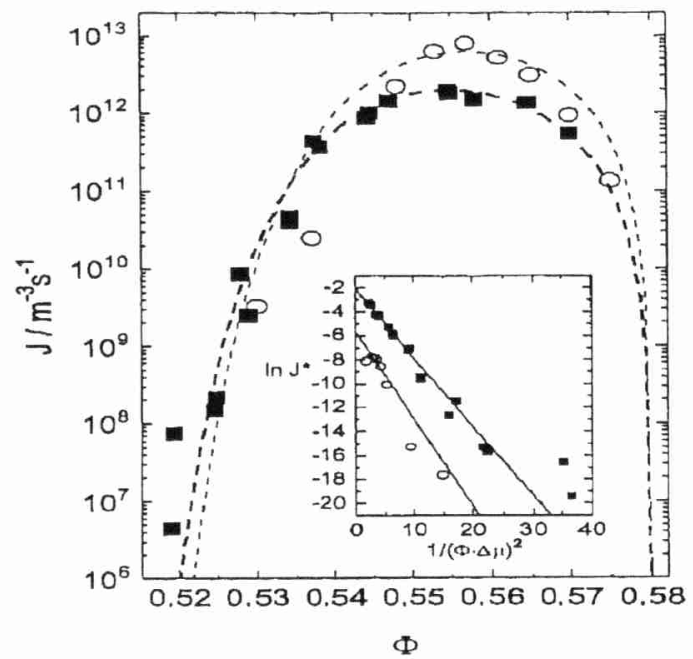

Fig. 3. Measured crystal nucleation rates $I$ as of function of volume fraction $\phi$ in a system of hard-sphere colloids. The data are taken from Ref. [5] (open circles) and Ref. [6] (filled cubes). The lines result from a two parameter fit of Eq. (4) to the experimental data. The inset shows the dimensionless nucleation rate densities plotted logarithmically versus $1 /(\phi \Delta \mu)^{2}$. The figure is taken from Ref. [4]

and obtained $\gamma=0.5 k_{B} T / \sigma^{2}$ and $\lambda=17 d_{N N}$, while for the data from Heymann et al. [6] he found $\gamma=0.54 k_{B} T / \sigma^{2}$ and $\lambda=2.8 d_{N N}$, where $\sigma$ is the particle diameter and $d_{N N}$ is the nearest neighbor distance. The estimates for the surface free energy are rather low when compared to numerical estimates [7] and the values of the effective jump length $\lambda$ seem rather large (a factor 10 to 100 larger than the mean free path in the liquid). However, as the experimental results could be fitted with Eq. (4), there was little reason to doubt the values of the fit parameters thus obtained from experiment.

As experiments to determine absolute crystal nucleation rates are notoriously difficult, there is a clear need for a first principle prediction of a crystal nucleation rate. 
In this review we discuss some of the recent progress that has been made in the application of computer simulation to gain a better understanding of the kinetics of colloidal crystallization.

\section{2}

\section{Method}

Simulating the crystallization process is a computational challenge, precisely because crystal nucleation is an activated process. This implies that the formation of small crystal nuclei in a supersaturated liquid is infrequent but, when it happens, the process is quite fast, i.e. it proceeds on a time scale that can be followed in a molecular simulation. For instance, experimentally measured nucleation rates are typically on the order of $O\left(10^{1}\right)$ to $O\left(10^{6}\right)$ nuclei per $\mathrm{cm}^{3}$ per sec. We can estimate the number of time steps needed in a molecular dynamics (MD) simulation to observe one nucleation event. In a large-scale computer simulation, it is feasible to study the dynamics of $O\left(10^{6}\right)$ particles, but the number of particles in a typical simulation is some two to three order of magnitude less. For an atomic liquid, the volume of a simulation box containing one million particles is of order $O\left(10^{-15}\right) \mathrm{cm}^{3}$. If a million nuclei form per second in one cubic centimeter, then it will take, on average, $10^{9}$ seconds for a nucleus to form in a system of a million particles. As the typical time step in a molecular simulation (MD) is on the order of femto seconds, this implies that it would take some $10^{24} \mathrm{MD}$ time-steps to observe a single nucleation event under experimental conditions.

This example illustrates why it will be difficult to compute nucleation rates using conventional MD simulations. One way around this problem is to simulate a system at a much higher supersaturation, where the free energy barrier for the formation of crystal nuclei is sufficiently low to allow the system to crystallize spontaneously on a time scale that is accessible in a MD simulation. The problem with this approach is that, at such extreme supersaturations, crystallization may proceed differently than at moderate supersaturations. For example at high supersaturations, many crystal nuclei may form simultaneously and may interact in an early stage of their development. It then becomes difficult to compare the computed crystallization rates with predictions based on CNT.

In order to study crystal nucleation at moderate supersaturation, we exploit the fact that the crystallization rate is determined by the product of a static term, namely the probability for the formation of a critical nucleus $P_{c}$, and a kinetic factor $\Gamma$ that describes the rate at which such nuclei grow. We use umbrella sampling to compute $P_{c}$ and kinetic Monte Carlo simulations to compute $\Gamma$. The computed nucleation rates can be directly compared to experimental data.

In the following we describe the numerical techniques needed to compute a nucleation rate based on Ref. [8]. First we discuss the calculation of the cluster size distribution. After that we turn to the calculation of the kinetic prefactor. 


\section{1}

\section{Calculation of the Cluster Size Distribution}

The probability to form a crystal nucleus of size $n$ can be approximated by $P(n)=$ $N_{n} / N$, where $N_{n}$ is the number of crystal nuclei of size $n$ in a system containing $N$ particles $[8,9,10]$, see also Appendix A. The approximation becomes better as $N_{n} / N$ becomes smaller, i.e. when the spontaneous formation of clusters is rare. Knowledge of the ratio $N_{n} / N$ allows us to define the Gibbs free energy $\Delta G(n)$ for the formation of a nucleus of size $n$ :

$$
\frac{N_{n}}{N}=\exp \left[-\Delta G(n) / k_{B} T\right]
$$

Before we can calculate $N_{n}$ in a Monte Carlo simulation we need to have a numerical technique that enables us to distinguish between particles in a liquid and solid environment. To this end, we use local bond-order analysis introduced by Steinhardt et al. [11] and applied to study nucleation by Frenkel and coworkers $[8,12,13]$. The advantage of this analysis is that it is only sensitive to the overall degree of crystallinity in the system, but independent of any specific crystal structure. This requirement is important as otherwise we would apply an external biasing potential, that could force the system to crystallize in a specific structure. A second advantage is that these bond-order parameters can be constructed so as to be independent of the reference frame.

The local bond-order parameters are a measure of the local structure around a particle and are constructed as follows. First we define a $(2 l+1)$ dimensional complex vector with the components

$$
q_{l m}(i)=\frac{1}{N_{b}(i)} \sum_{j=1}^{N_{b}(i)} Y_{l m}\left(\hat{\mathbf{r}}_{i j}\right),
$$

where the sum goes over all neighboring particles $N_{b}(i)$ of particle $i$. Neighbors are usually defined as all particles that are within a given radius $r_{q}$ around a particle. $Y_{l m}\left(\hat{\mathbf{r}}_{i j}\right)$ are the spherical harmonics evaluated for the normalized direction vector $\hat{\mathbf{r}}_{i j}$ between the neighbors.

The orientation of the unit vector $\hat{\mathbf{r}}_{i j}$ is determined by the polar and azimuthal angles $\theta_{i j}$ and $\phi_{i j}$. The rotationally invariant local bond-order parameters are then defined as follows:

$$
q_{l}(i)=\left(\frac{4 \pi}{2 l+1} \sum_{m=-l}^{l}\left|q_{l m}(i)\right|^{2}\right)^{1 / 2}
$$

and

$$
\hat{w}_{l}(i)=\frac{w_{l}(i)}{\left(\sum_{m=-l}^{l}\left|q_{l m}(i)\right|^{2}\right)^{3 / 2}}
$$

with 

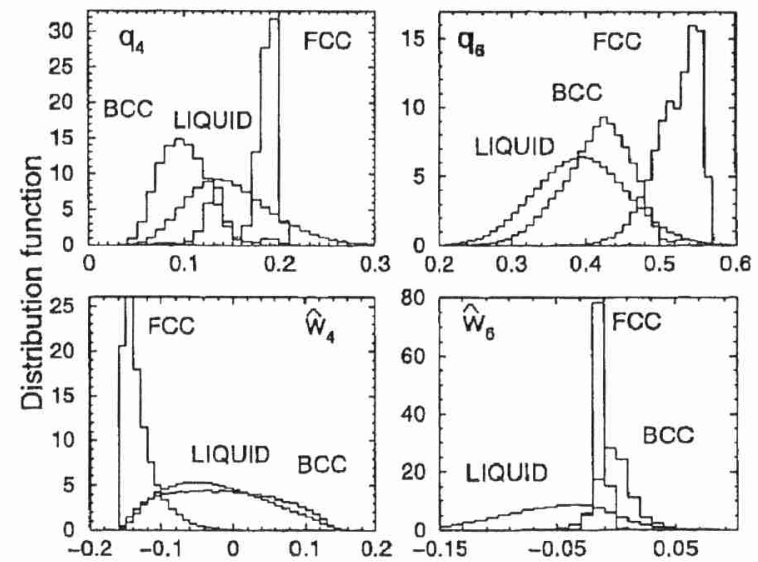

Fig. 4. Distribution functions of the local bond-order parameters: $q_{4}, q_{6}, \hat{w}_{4}$ and $\hat{w}_{6}$ from Monte Carlo simulations in a hard-sphere system. Here the cutoff radius $r_{q}$ for the local environment of a particle is chosen to be $1.4 \sigma$, where $\sigma$ is the hard-core diameter. This means that we included the first, and in some cases also the second nearest neighbors

$$
w_{l}(i)=\sum_{\substack{m_{1}, m_{2}, m_{3} \\
m_{1}+m_{2}+m_{3}=0}}\left(\begin{array}{ccc}
l & l & l \\
m_{1} & m_{2} & m_{3}
\end{array}\right) q_{l m_{1}}(i) q_{l m_{2}}(i) q_{l m_{3}}(i) .
$$

The term in brackets in the last equation is the Wigner-3j symbol. In Fig. 4 we show typical distribution functions of the local bond-order parameters $q_{4}, q_{6}, \hat{w}_{4}, \hat{w}_{6}$ calculated in a Monte Carlo simulation of hard-spheres under conditions close to the coexistence point, where the liquid and the solid phase are equally stable. The figure illustrates that there is some separation between the distribution functions obtained from the liquid and those obtained from the solid. Sometimes, there is even a separation between the solid structures themselves, a property that we will use later to distinguish between different solid structures. For the identification of solid-like particles we have to choose an order parameter that is able to distinguish between the liquid on the one hand, and all possible solid structures, on the other. From Fig. 4 we see that $q 6$ has some of the desired properties, as the values of the solid phases are all shifted to higher values compared to the liquid. These order parameters are sensitive to the degree of orientational correlations of the vectors that join neighboring particles. In simple liquids we expect that there are no preferred orientations around a particle and therefore the correlations decay rapidly. In contrast, for a particle with a solid-like environment the vectors are correlated and as result there should be a clear separation between distribution functions for the bond-order parameter. For this reason we can enhance the selectivity of the method by calculating the correlation function of the vectors $\mathbf{q}_{6}$ of neighboring particles $i$ and $j$

$$
\mathrm{q}_{6}(i) \cdot \mathrm{q}_{6}(j)=\sum_{m=-6}^{6} q_{6 m}(i) \cdot q_{6 m}^{*}(j),
$$


where the * indicates the complex conjugate. In Fig. 5 we show the corresponding distribution functions for a hard-sphere system. Note that we did not attempt to normalize the dot-product. The relevant solid structures, which for the hard sphere system are fcc, hcp and bcc, yield much higher values for the dot-product than the liquid. We now define two neighboring particles $i$ and $j$ to be connected, if the dotproduct described above exceeds a certain threshold. In the case of hard spheres this threshold is set to 20. By using this definition we can correctly identify effectively all particles in a solid to be solid-like, however also in the liquid it happens quite frequently that a particle has more than one connection. To illustrate this, we show in Fig. 6 the distribution functions for the number of connections per particle. Note, that the peak for the solid structures is at $12 \mathrm{for} f c c$, hcp and around 13 for bcc. These numbers correspond to the first, or first and second nearest neighbors, which were included in the local environment. For the bcc structure the peak is slightly shifted to lower values, which is due to the fact that the bcc structure is relatively disordered. The bcc lattice of monodisperse hard-spheres melts spontaneously. We found, however, that a slightly polydisperse (3\%) bec crystal is mechanically stable. We used such a crystal to study the bcc bond-order properties. Thus far, we have no clear separation between solid-like and liquid-like particles, because the order-parameter distributions overlap. We therefore apply a more stringent criterion to distinguish between solid and liquid. To this end, we impose a threshold on the number of connections a particle has with its neighbors. All particles with less connections than this threshold are considered to be liquid-like. We should bear in mind that, in a small nucleus, most particles are at the surface. These should be recognized as solid-like. We found this is achieved if we choose threshold value between 6 and 8 . The present analysis provides us with an unambiguous local criterion to identify solid-like particles. Finally, we need a criterion to identify which solid particles belong to a single cluster. For this purpose, we used a simple distance criterion: if two solid-like particles are closer than a certain threshold distance, then they belong to the same cluster. The values that we chose for this were between $1.5 \sigma$ and $2 \sigma$, where $\sigma$ is the hardcore diameter. We note that, whereas the absolute number of particles in the cluster depends somewhat on the choice of the threshold values, the height of the computed free-energy barriers is fairly insensitive to the precise criterion that is used.

Using this local bond-order analysis we can sample the equilibrium distribution function for the probability $P(n)$ in a Monte Carlo simulation. In all cases we performed Monte Carlo simulations in the isobaric-isothermal (NPT) ensemble. In this ensemble the average of a microscopic quantity $A$ is given by

$$
\langle A\rangle_{N P T}=\frac{\int d V \int d \mathbf{r}^{N} A\left(\mathbf{r}^{N}\right) \exp \left[-\beta\left(U\left(\mathbf{r}^{N}\right)+P V\right)\right]}{\int d V \int d \mathbf{r}^{N} \exp \left[-\beta\left(U\left(\mathbf{r}^{N}\right)+P V\right)\right]},
$$

where $U\left(\mathbf{r}^{N}\right)$ is the potential energy of the system with particle positions $\mathbf{r}^{N} \cdot \beta=$ $1 / k_{B} T$ is the reciprocal of the thermal energy, $N$ the number of particles and $P$ the applied pressure. In a Metropolis Monte Carlo simulation the above ensemble average is approximated by 


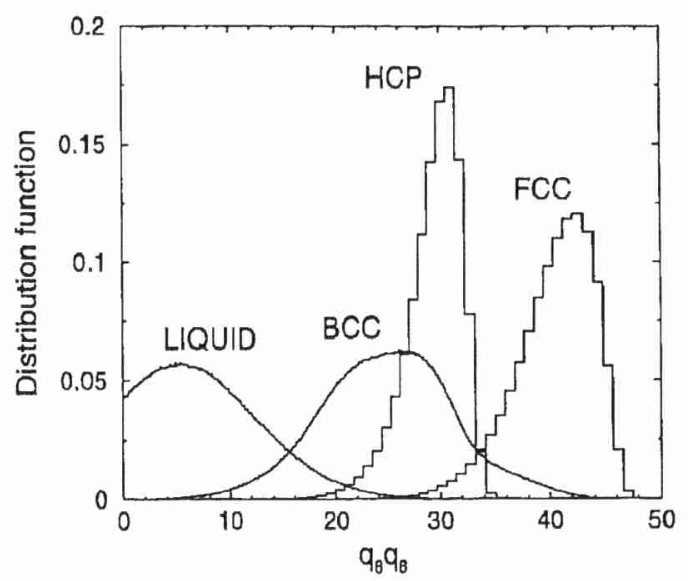

Fig. 5. Distribution functions of the dot product $\mathrm{q}_{6}(i) \cdot \mathrm{q}_{6}(j)$ from Monte Carlo simulations in a hard-sphere system

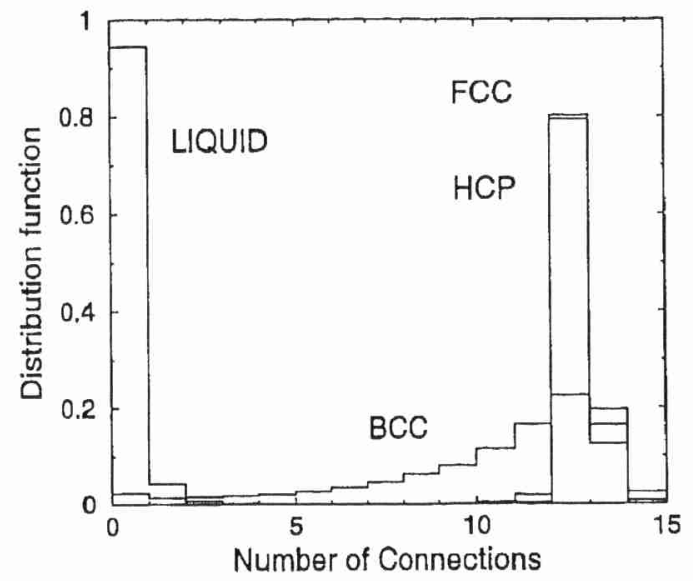

Flg. 6. Distribution functions of the number of connections per particle from Monte Carlo simulations in a hard-sphere system

$$
\langle A\rangle_{N P T} \approx \frac{1}{M} \sum_{i=1}^{M} A\left(\mathbf{r}_{i}^{N}\right),
$$

where $M$ is the total number of measurements and $A\left(\mathbf{r}_{i}^{N}\right)$ the value of our property $A$ associated with configuration $r_{i}{ }^{N}$. In the case of crystal nucleation we need to calculate the average number of clusters of size $n$ and Eq. (8) becomes

$$
\left\langle N_{n}\right\rangle_{N P T} \approx \frac{1}{M} \sum_{i=1}^{M} N_{n}\left(\mathrm{r}_{i}{ }^{N}\right) .
$$


As an example we show the results from Monte Carlo simulations in a system of hard-spheres. In the simulations we used $N=3375$ particles and applied a pressure $\beta P \sigma^{3}=16$. At this pressure, the liquid phase is meta stable with respect to the solid, but does not crystallize spontaneously as the Gibbs free energy barrier between the two states is too high. The temperature $T$ does not play a role in that system. After equilibrating the system, one could in principle measure the cluster size distribution after every Monte Carlo move, however this would be computationally expensive and statistics would still be poor, as the measurements are strongly correlated. Instead we measure the cluster size distribution after one trajectory, which consists of 20 moves per particle plus about 10 volume moves. The total length of the simulation was 100000 trajectories. In this simulation we could measure the probability distribution $P(n)$ up to cluster sizes of $n=15$ particles. The corresponding Gibbs free energy for the formation of such a cluster is shown in the inset of Fig. 8. The formation of larger cluster was so rare that the statistical accuracy was too poor.

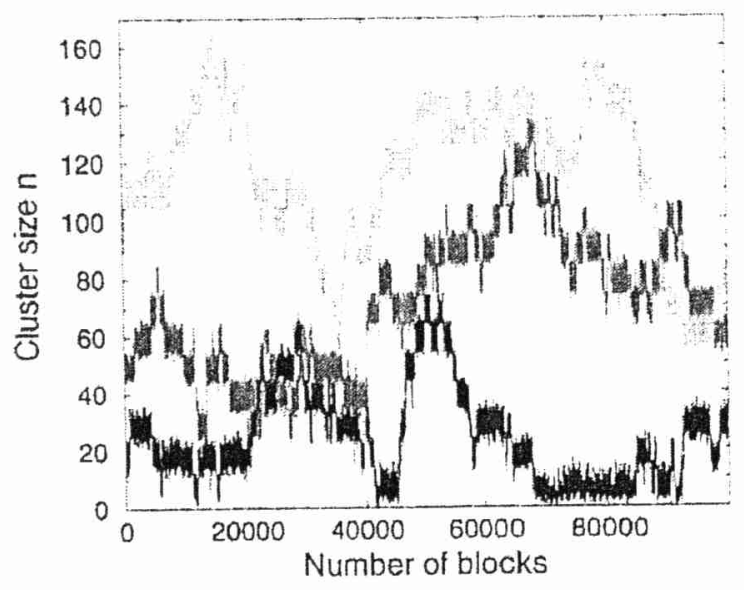

Fig. 7. Examples of the cluster size sampled during one simulation. The different configurations started with clusters of sizes $n=20,50$ and 110 . Due to the parallel tempering technique, swapping between different windows is possible and the configurations could sample almost all possible cluster sizes

In order to sample larger cluster sizes we needed to apply the umbrella sampling technique of Torrie and Valleau [14]. The method is based on the idea that the ensemble average can be rewritten as follows

$$
\langle A\rangle_{N P T}=\frac{\left\langle A /\left.W\left(\mathbf{r}^{N}\right)\right|_{W}\right.}{\left\langle W\left(\mathbf{r}^{N}\right)^{-1}\right\rangle_{W}},
$$

where we have introduced an, as yet unspecified, weighting function $W\left(\mathrm{r}^{N}\right)=$ $\exp \left[-\beta \omega\left(\mathbf{r}^{N}\right)\right]$, where $\omega\left(\mathbf{r}^{N}\right)$ is the biasing potential. The subscript $\langle\ldots\rangle_{W}$ indicates 
an ensemble average according to the biased distribution function $\exp \left[-\beta\left(U\left(\mathrm{r}^{N}\right)+\right.\right.$ $P V)] W\left(\mathbf{r}^{N}\right)$. By specifying the weighting function $W$ we can force the system to sample in the relevant regions of phase space. In the case of crystal nucleation we can calculate the ensemble average according to the weighted ensemble, Eq. (9), as follows

$$
\left\langle N_{n}\right\rangle_{N P T} \approx \frac{\sum_{i}^{M}\left[N_{n}\left(\mathbf{r}_{i}^{N}\right) / W\left(\mathbf{r}_{i}^{N}\right)\right]}{\sum_{i}^{M}\left[W\left(\mathbf{r}_{i}^{N}\right)^{-1}\right]}
$$

where the sum goes over all measurements $M$. We now need to consider the choice of the weighting function. As the formation of large nuclei is rare, the probability to have two large clusters simultaneously in the system, is vanishingly small. As a consequence, we can choose a bias potential that just controls the size of the largest cluster in the system. Somewhat arbitrarily, we chose the bias potential to be a harmonic function of the size of the largest cluster:

$$
\omega\left[n\left(\mathbf{r}^{N}\right)\right]=\frac{1}{2} k_{n}\left[n\left(\mathbf{r}^{N}\right)-n_{0}\right]^{2} .
$$

The constant $k_{n}$ determines the range of cluster-sizes sampled in one simulation. The parameter $n_{0}$ determines the center of the "window". In principle, it should be possible to design a biasing function that makes it possible to sample all cluster sizes in a single simulation. However, such a "smart" simulation would take much longer to equilibrate [15]. This is why we split the simulation into a number of smaller simulations that are restricted to narrow, but overlapping windows of different cluster sizes. The implementation of the biasing potential in the Monte Carlo simulation is straightforward. As the computation of cluster sizes is relatively time-consuming, we do not compute the size of the largest cluster after every Monte Carlo move. Rather, we carry out a fixed number of Monte Carlo moves per particle without bias. We then calculate the final cluster size and accept or reject the whole sequence of trial moves on basis of the change in the biasing potential: $\exp [-\beta \Delta \omega]$, where $\Delta \omega$ is the difference in the biasing potential after and before the trajectory. To facilitate the (very slow) stacking rearrangements of the clusters, we implemented the parallel tempering scheme of Geyer and Thompson [16]. The idea is to run all the simulations in the different windows in parallel and allow them to exchange clusters between adjacent windows. The actual change between windows $i, j$ is accepted according to $\exp \left[-\beta\left(w_{n}-w_{o}\right)\right]$, where $w_{o}=k_{i} / 2\left(n_{i}-n_{0, i}\right)^{2}+k_{j} / 2\left(n_{j}-n_{0, j}\right)^{2}$ is the energy of the biasing potential before and $w_{n}=k_{i} / 2\left(n_{j}-n_{0, i}\right)^{2}+k_{j} / 2\left(n_{i}-n_{0, j}\right)^{2}$ after the change. In practise, what is exchanged between processors, are the minima of the bias potential rather than configurations. This requires virtually no communication between different computer nodes. In Fig. 7 we show an example of the cluster sizes sampled in the course of a simulation of hard spheres.

In the inset of Fig. 8 we show the results for the Gibbs free energy of a nucleus obtained from the simulations in each window (unbiased+biased runs). The Gibbs free energies in the different windows are determined up to a constant $\Delta G_{i}(n) / k_{B} T+b_{i}$, where the subscript $i$ indicates the number of the window. In order to determine the 


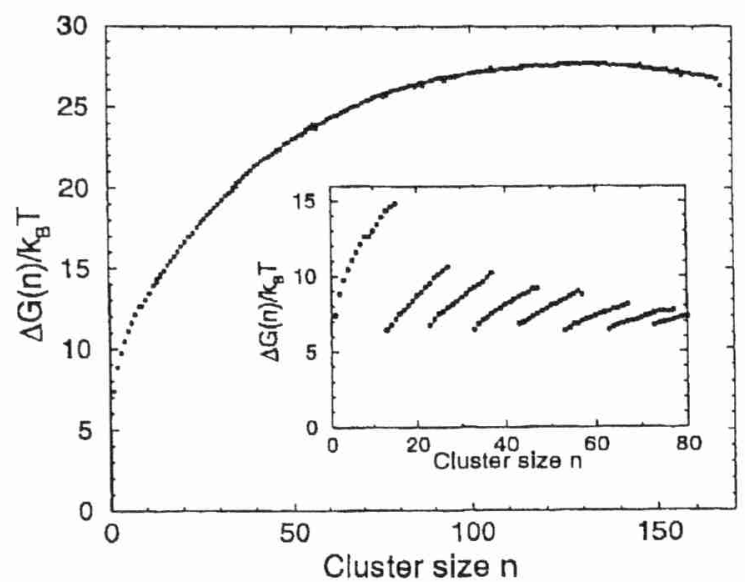

Fig. 8. Gibbs free energy for the formation of a cluster of $n$ hard-spheres at $\beta P \sigma^{3}=16$, after fitting the results for the free energy in the different windows to one polynomial. The inset shows the sequence (unbiased + biased) of measured Gibbs free energies $\Delta G_{i}(n) / k_{B} T+b_{i}$ before the fit

constants $b_{i}$ we fitted all the free energy estimates in the different windows to one polynomial in $n$. This can be done by a linear least-square fit, where we minimize

$$
\chi=\sum_{n=1}^{n_{\max }}\left\{\sum_{i=1}^{n_{10}} w_{i}(n)\left[\Delta G_{i}(n)-\sum_{k=1}^{k_{\max }} a_{k} n^{k}-b_{i}\right]^{2}\right\} .
$$

Here $w_{i}(n)=1 / \sigma_{\Delta G_{i}(n)}^{2}$ is the statistical weight determined by the variance $\sigma_{\Delta G_{i}(n)}^{2}$ of the free energy measurement and $n_{w}$ the total number of windows used in the simulation. The maximum order of the polynomial used was $k_{\max }=10$. Note that by using a high-order polynomial, we do not assume a functional form of the nucleation barrier (the barrier might or might not be correctly described by CNT). From the unbiased simulation we get the absolute Gibbs free energy for the formation of a cluster of size $n$ with respect to the liquid state. Therefore the constant $b_{1}$ is known. In Fig. 8 we show the final result for the calculation of a nucleation barrier for hardspheres at pressure $\beta P \sigma^{3}=16$.

\section{2}

\section{Kinetic Prefactor}

In atomistic simulations, the kinetic prefactor is usually calculated using the BennettChandler scheme [17]. In the case where the barrier crossing is relatively diffusive, it is attractive to use a modification proposed by Ruiz-Montero et al. [18]. The principle of both methods is to generate a large number of independent configurations at the top of the barrier. These configurations are then used as the starting point for an unbiased trajectory in which one determines if the nucleus grows and the system 
crystallizes, or if it shrinks. From the number of nuclei that grow and shrink one can extract the kinetic factor. However, in order to get a reasonable estimate one has to simulate a rather large number of trajectories, on the order of one hundred. In the present work, we consider barrier crossing phenomena that are, effectively, purely diffusive. In that case, we can compute the kinetic prefactor directly using the expression: $\Gamma=Z_{p_{l}} f_{n_{c}}^{+}$. After a barrier calculation at number density $\rho_{i}$ the only unknown quantity is $f_{n_{c}}^{+}$. In order to compute $f_{n_{c}}^{+}$, we assume that the critical cluster grows and shrinks via the diffusive attachment of single particles. We can then define an effective diffusion constant for the change in critical cluster size:

$$
D_{n_{c}}^{a \| t}=\frac{1}{2} \frac{\left\langle\Delta n_{c}^{2}(t)\right\rangle}{t} .
$$

Here $\Delta n_{n_{c}}^{2}(t)=\left[n_{c}(t)-n_{c}(t=0)\right]^{2}$ is the mean square change in the number of particles in the critical cluster. As the slope of this change is related to the corresponding attachment rates via $\left\langle\Delta n_{c}^{2}(t)\right\rangle / t=\left(f_{n_{c}}^{+}+f_{n_{c}}^{-}\right) / 2$, and as we know that, at the top of the barrier, the forward and backward rates are equal $\left(f_{n_{c}}^{+}=f_{n_{c}}^{-}\right)$, we get

$$
f_{n_{c}}^{+}=\frac{1}{2} \frac{\left\langle\Delta n_{c}^{2}(t)\right\rangle}{t}
$$

This is a general expression for the calculation of the kinetic factor for diffusive barrier crossing. Using a Molecular Dynamics simulation one only needs to measure the change in size of the critical cluster as a function of time. The only restriction is that, during the measurement, the critical nucleus needs to fluctuate around its critical value.

To apply this method for the calculation of the attachment rate in a colloidal suspension, we need to have a simulation technique that generates trajectories following Brownian dynamics and hydrodynamic interaction also needs to be considered. Trajectories following Brownian dynamics could be generated using a kinetic Monte Carlo scheme proposed by Hinson and Cichocki [19]. These authors show that, in the limit of very small maximum particle displacement, $\Delta x_{\max } \rightarrow 0$, the trajectories generated by the kinetic Monte Carlo simulation are stochastically equivalent to the process described by the Smoluchowski equation. The limit $\Delta x_{\max } \rightarrow 0$ means that simulation time would become infinitely long. However, Hinson and Cichocki also propose an extrapolation procedure with which this limit can be approached systematically by repeating simulations with a smaller maximum displacement. In experiments nucleation rates are usually presented in dimensionless form $I^{*}=I k \sigma^{5} / D_{0}$, where $\sigma$ is the diameter of a monomer and $D_{0}$ the free diffusion coefficient. Therefore we only need to compute the ratio $f_{n_{c}}^{+} / D_{0}$. From the previous calculation of the nucleation barrier in a hard-sphere system we could determine the critical cluster size and had generated independent configurations in which such a cluster was stabilized. We used these configurations, to perform an unbiased kinetic NVE Monte Carlo simulation, measuring the size of the critical cluster as a function of Monte Carlo blocks. Here one block is 100 trial moves per particle. In the inset of Fig. 9 we show such a measurement at $\phi=0.5277(P=16)$. From these data we then extracted the 


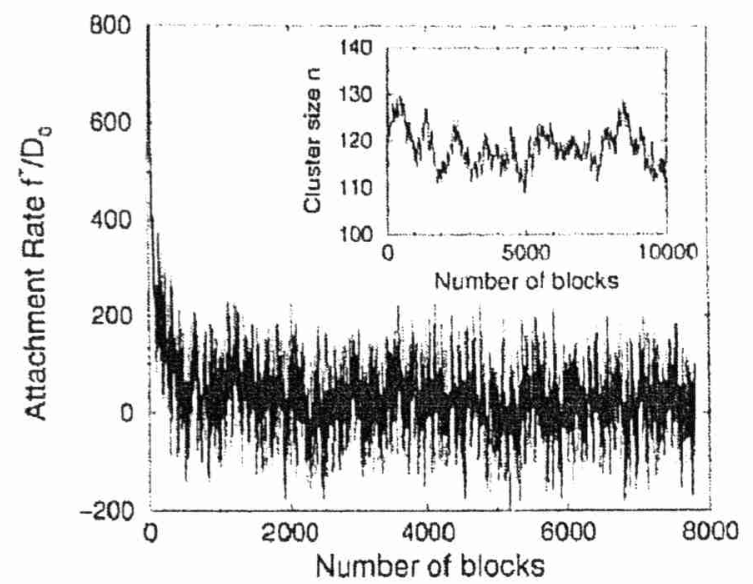

Fig. 9. Reduced attachment rate $f_{n_{c}}^{+} / D_{0}$ of particles to the critical cluster at volume fraction $\phi=0.5277$. Here one block is 100 moves per particle in an NVE Monte Carlo simulation. The inset shows the development of the size $n$ of the critical cluster during one trajectory

attachment rate using Eq. (12) which is shown in the same figure. Surprisingly, we see that the attachment rate has a different short time and long time behavior. This implies that, at short times, the diffusion in cluster size is not a Markov process. As the diffusion of the critical cluster over the nucleation barrier is on the time scale of the long time behavior of the attachment rate, this is the value we have to use. To test the dependence of our results on the maximum particle displacement we performed simulations for two different values $\Delta x_{\max }=0.12 \sigma$ and $0.012 \sigma$. The corresponding values for the free diffusion coefficients are $D_{0}=\left\langle\Delta x_{\max }^{2}\right\rangle / 6$. The ratio of the results for $f_{n_{c}}^{+} / D_{0}$ in both simulations is equal to 4.79. Computing the long time self diffusion coefficient $D_{S}^{L} / D_{0}=\left\langle(r(0)-r(t))^{2}\right) / 6 t D_{0}$ we get a ratio in both simulations of 5.07. Therefore the difference in the results for the attachment rate is mainly due to diffusion. In our simulations we did not follow the extrapolation procedure for $\Delta x_{\max } \rightarrow 0$ described in [19], as for $\Delta x_{\max }=0.012 \sigma$ we are already in a limit where the attachment rate has effectively reached its limiting value. We justify this by testing our approach on the calculation of the long time self diffusion coefficient, which will be discussed later.

To correct for the effect of hydrodynamic interactions that are known to be important at high volume fractions, we used an approach proposed by Medina-Noyola [20]. To this end, we replace the free diffusion coefficient $D_{0}$ by the short-time self diffusion coefficient $D_{S}^{S}$. We therefore have to multiply our result by a factor $\alpha=D_{S}^{S} / D_{0}$. In the case of hard spheres, several (rather similar) functional forms for this factor have been proposed in the literature $[21,22,23,24]$. Here we used the phenomenological expression $(1-\phi / 0.64)^{1.17}[25]$ at high volume fraction $\phi$. As a test of our approach we computed the long-time self diffusion coefficient of a dense colloidal suspension of hard spheres. Our results, $D_{S}^{L} / D_{0}=2.9 \times 10^{-3}, 2.5 \times 10^{-3}, 2.1 \times 10^{-3}$, 
calculated at volume fractions $\phi=0.5207,0.5277,0.5342$, are within statistical error of experimental data, see e.g. [26, 39].

For the calculation of the kinetic factor we usually performed about 5 trajectories. The length of the trajectory depends on whether the cluster size fluctuates around the critical size or not; if not the simulation is stopped. From these simulations we calculated the attachment rate. The error estimates vary between a factor of one for the larger critical cluster sizes and a factor of two to three for the smaller cluster sizes. In the regime of smaller critical cluster sizes, the fluctuations in cluster size are almost on the order of the critical cluster size and it becomes therefore more difficult to get a good estimate.

\section{3}

\section{Hard-Sphere Colloids}

A collection of hard, identical spheres is the simplest possible model system that undergoes a first order phase transition. For low packing fractions the particles are in a liquid state, but when the packing fractions exceeds a value of $49.4 \%$ a ordered solid state becomes more stable. This was first shown in computer simulations by Hoover and Ree [27] in 1968. The experimental realization of a colloidal suspension that closely mimics the phase behavior of hard spheres followed about 20 years later and was a milestone in soft matter physics [28, 29]. More recently the phase transition kinetics of hard sphere colloids has been studied extensively in experiments $[5,30,31]$. However as mentioned in the introduction the interpretation of the data with CNT was rather indirect.

Using the simulation techniques described before, we can compute the rate of crystal nucleation for hard sphere colloids by a direct calculation of the nucleation barrier and the kinetic prefactor [8, 32]. We first performed Monte Carlo simulations in the isobaric-isothermal ensemble $N P T$ to compute the crystal nucleation barrier at three different pressures $\beta P \sigma^{3}=15,16,17$. The corresponding bulk volume fractions of the liquid are $\phi=0.5207,0.5277,0.5343$. The resulting nucleation barriers are shown in Fig. 10. As expected, with increasing volume fraction the crystal nucleation barrier decreases. Our simulation results for the crystal nucleation barrier can be compared directly to the predictions from CNT for the nucleation barrier Eq. (1). For the hard-sphere system the chemical potential difference can be calculated accurately using phenomenological equations of state for the liquid and the solid [33], see Appendix B. As the solid-liquid interfacial free energy $\gamma$ of a small crystal nucleus in a supersaturated liquid is not known a priory we use its corresponding value for a flat interface at coexistence. This value has been calculated in a recent simulation [7] for three different crystal planes. Here we use $\gamma_{a v}=0.61 k_{B} T / \sigma^{2}$ which is the average of the three crystal planes. The results for the barrier height based on CNT in order of increasing density are $\Delta G^{*} / k_{B} T=27,15.7,10.2$. These values are about $30-50 \%$ lower than our numerical estimate. This discrepancy might be due to the fact that for a small nucleus in a supersaturated liquid the interfacial free energy is different from that of a flat interface at coexistence. For this reason we also used $\gamma$ 


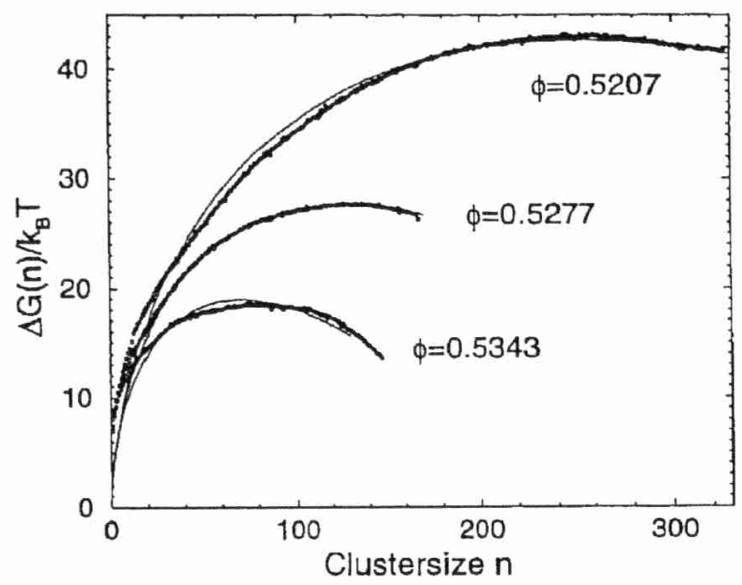

Fig. 10. Calculated free energy barrier for homogeneous crystal nucleation of hard-sphere colloids. The results are shown for three values of the volume fraction. The drawn curves are fits to the CNT-expression Eq. (1). For the identification of solid like particles we used the techniques described before. The cutoff for the local environment was set to $r_{q}=1.4 \sigma$, the threshold for the dot product $9696=20$ and the threshold for the number of connections was set to 6 . If two solidlike particles are less than $2 \sigma$ apart, where $\sigma$ is the diameter of a particle, then they are counted as belonging to the same cluster. The total simulation was split up into a number of smaller simulations that were restricted to a sequence of narrow, but overlapping, windows of $n$ values. The minimum of the bias potential was placed in steps of tens, i.e $n_{0}=10,20,30, \ldots$ In addition we applied the parallel tempering scheme of Geyer and Thompson [16] to exchange clusters between adjacent windows. All simulations were carried out at constant pressure and with the total number of particles (solid plus liquid) fixed. For every window, the simulations took at least $1 \times 10^{6} \mathrm{MC}$ moves per particle, excluding equilibration. To eliminate noticeable finite-size effects, we simulated systems containing 3375 hard spheres. We also used a combined Verlet and Cell list to speed up the simulations

as a fit parameter to our results. Using $R=\left(3 n /\left(4 \pi \rho_{S}\right)^{1 / 3}\right.$ we fitted Eq. (1) to our data. The result can be seen as the solid line in Fig. 10. As can be seen, the functional form of the nucleation barrier seems to be described well by CNT, but the values for the fit parameter $\gamma_{\text {eff }}(P=15)=0.71 k_{B} T / \sigma^{2}, \gamma_{\text {eff }}(P=16)=0.737 k_{B} T / \sigma^{2}$ and $\gamma_{\text {eff }}(P=17)=0.751 k_{B} T / \sigma^{2}$ are higher than the coexistence value and they increase with volume fraction. If we assume that this dependence is linear, than our simulation results extrapolate to a value of $\gamma_{\text {eff }}(P=11.7)=0.64 k_{B} T / \sigma^{2}$ at coexistence $-\mathbf{a}$ value that is very close to $\gamma_{a v}$. For a discussion of the dependence of the surface free-energy density on supersaturation, see Ref. [34]. In Appendix C we discuss an alternative, thermodynamic route to compute the surface free-energy density for the critical nucleus.

Our results for the surface free-energy density can also be compared to the values extracted from experiments. Palberg [4] fitted the data from Harland and van Megen [5] and obtained $\gamma=0.5 k_{B} T / \sigma^{2}$ and for the data from Heymann et al. [6] 
he found $\gamma=0.54 k_{B} T / \sigma^{2}$. Note that these values are significantly lower than the numerical estimates.

In the crystal-nucleation experiments, the colloids had a size polydispersity of about $5 \%$. We therefore repeated our simulations for a suspension with $5 \%$ polydispersity. We find that both systems have the same nucleation barrier at the same $\Delta \mu$ [35]. For a caculation of $\Delta \mu$ see Appendix C. Therefore polydispersity alone cannot account for the difference between the barrier heights derived from experiment and simulation.

Table 1. Summary of the simulation results for the calculation of the nucleation rate for monodisperse hard sphere colloids. Here $\phi$ is the volume fraction of the liquid phase. $\Delta G^{*}$ is the measured free energy to form a cluster of critical size $n_{c} . f_{c}^{+} / D_{0}$ is the attachment rate of particles to the critical cluster divided by the free diffusion coefficient. $I^{*}=I \sigma^{5} / D_{0}$ is the reduced nucleation rate, and $\lambda$ is the estimated typical jump distance from the calculation of the attachment rate. $\Delta \mu$ is the difference in chemical potential between the two phases

\begin{tabular}{ccccccc}
\hline$\phi$ & $\Delta G^{*}$ & $n_{c}$ & $f_{c}^{+} / D_{0}$ & $\log _{10}\left[I^{*}\right]$ & $\lambda$ & $\Delta \mu$ \\
\hline 0.5207 & 43.0 & 260 & 189 & -19.3 & 0.31 & 0.34 \\
0.5277 & 27.8 & 130 & 43 & -13.5 & 0.46 & 0.44 \\
0.5342 & 18.5 & 75 & 66 & -9.14 & 0.27 & 0.54 \\
\hline
\end{tabular}

Subsequently, we performed kinetic Monte Carlo simulations to compute the kinetic prefactor and, thereby, the absolute crystal nucleation rate. The results of our calculations of the attachment rate for the monodisperse hard-sphere system are summarized in Table 1. As experimentally determined values for the kinetic factor often differ by orders of magnitude from those predicted by CNT, it is also important to compare our computed kinetic prefactor with the one predicted by CNT. We find that the Zeldovich factor that follows from our numerical calculations is almost identical to the CNT prediction. This is not surprising, as CNT provides a good fit to the numerical data for the shape of the barrier. The remaining quantity to compare is the reduced attachment rate $f_{c}^{+} / D_{0}$. If we assume that in Eq. (5), $D_{S}=D_{S}^{L}$, where $D_{S}^{L}$ is the long-time self-diffusion constant, and if we treat $\lambda$ as a fit parameter to reproduce our calculated attachment rates, then we get values for $\lambda$ in the range $0.27-0.46 \sigma$ (see Table 1). This jump distance - in the case of colloids it might be better to call it a diffusion distance - is comparable to the inter-particle spacing in a dense suspension, which seems reasonable. In contrast, experimental estimates for $\lambda$ tend to be rather large: $\lambda=2.8-17 \sigma$ [4]. The identification $D_{S}=D_{S}^{L}$ is justified by the fact that the time $\lambda^{2} / D_{S}^{L}$ corresponds to long-time diffusion.

Using our simulation results we can compute steady-state nucleation rates that can be compared directly (i.e. without any adjustable parameters) to experiment. In Fig. 11 we show our numerical predictions for the nucleation rate of a monodisperse suspension and a suspension with $5 \%$ polydispersity. These results can be compared directly to the experiments on suspensions with the same polydispersity. Note that 
the polydispersity in Ref. [31] is about $2.5 \%$. As can be seen from the figure, the simulations predict a much stronger dependence of the nucleation rates on density than is observed in the experiments. This discrepancy between the simulations and experiment is unexpected and significant because hard-sphere colloids are among the best studied experimental realizations of a simple liquid. We know the structural and thermodynamic properties of hard-sphere suspensions quite accurately and, more significantly, these properties tend to be well reproduced by the ideal, hard-sphere model. Hence, large discrepancies between experiment and simulation cannot be easily dismissed as being due to uncertainties in the parameters that characterize the colloidal suspension. Rather, we must envision the possibility that either our theoretical description of crystallization is inadequate or that what is measured is not really the steady-state, homogeneous nucleation rate. In fact, the latter suggestion is not altogether unreasonable, as light-scattering cannot be used to see the very early stages of crystal nucleation. Secondly, the experiments are extremely sensitive to any residual ordering in the solution that may have survived the preparation of the experimental system. Thirdly, at high supersaturations, the concentration of crystal nuclei rapidly becomes sufficiently large that the interaction between different crystal nuclei may no longer be ignored [36]. In that case, the steady-state nucleation expressions that we employ are no longer applicable. We note that Dixit and Zukoski [37] developed a purely kinetic model to predict nucleation rates which yields good quantitative agreement with the experimental data. Volkov et al. [38] recently reported molecular dynamic simulations of hard-sphere crystallization at large supersaturations. In this regime, the simulations are in good agreement with experiment. In fact, in the simulations of Volkov et al., the simulation data could be analyzed in the same way as the experiments (namely, by studying the time evolution of the first Bragg peak of the static structure).

One unique feature of the simulations is that they allows us to study, in detail, the structure of small crystal nuclei. This is interesting as already in 1897 Ostwald [40] pointed out the role of meta-stable phases in crystal nucleation when he formulated his famous step rule. This rule states that the phase that nucleates does not need to be the one that is thermodaynamically most stable. In the recent years there have been several attempts to provide a microscopic explanation [41, 42, 43, 44] for Ostwald's observation. Alexander and McTague [41] argue, on the basis of Landau theory, that if the differences in the liquid and solid densities were not too great, then the phase that would be nucleated from the liquid would be bcc regardless of the structure of the stable (lowest free energy) phase. Leyvraz and Klein [42, 43], showed that for deeply quenched systems with long-range interactions, the critical droplet can have a bcc symmetry, though not a bcc crystalline structure. Simulations by ten Wolde et al. [13] showed that the situation can even be more subtle, at least for a LennardJones system: The core of a stable Lennard-Jones cluster formed a stable fcc structure while the surface of the nucleus showed indications of a bcc structure. Thermodynamically the formation of metastable phases might be explained by differences in interfacial free energies. The formation of a bcc-liquid interface might cost less energy than that of a fcc-liquid interface. In the case of hard-spheres it is known that the fcc phase is the stable structure, but the free energy difference between the fcc 


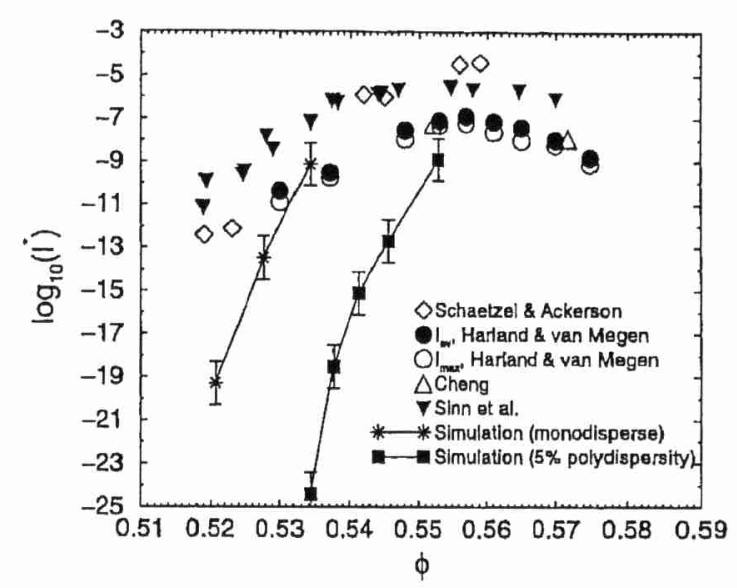

Fig. 11. Reduced nucleation rates $\left(I^{*}=I \sigma^{5} / D_{0}\right)$ as a function of the volume fraction of the meta-stable liquid. The simulation data for monodisperse colloids are indicated by the *the drawn curve joining the simulation points is meant as a guide to the eye. In the same figure we show the experimental results of Ref. [30] ( 0 ), Ref. [5] (o and $\bullet$ ), Ref. [39] $(\Delta)$ and Ref. $[31](\nabla)$. We also performed simulations on model systems that have the same polydispersity $(5 \%)$ as the experimental systems. These simulation results are denoted by the filled squares

and the hcp structure is very small $\left(<10^{-3} k_{B} T\right)[45,46]$. This means that thermal fluctuations of the order of $k_{B} T$ could transform a cluster of 1000 particles from fcc to hcp or just cause stacking faults. Note that the fcc and the hcp structure differ only in the stacking of close-packed hexagonal crystal planes. For the fcc structure the stacking is $A B C$, whereas for the hcp structure the stacking is $A B$. If the interfacial free energies of a crystal fcc-liquid, hcp-liquid or a rhcp-liquid interface are different, than this could also completely change this picture. Here rhcp refers to a random stacking of the close-packed hexagonal crystal planes. The question if small crystal nuclei are more fcc or hcp like is not clear. Experiments by Pusey et al. [47] and Elliot et al. [48] indicate that the fcc structure is favored. However, microgravity experiments by Zhu et al. [49] showed that, initially, small crystal nuclei have a rhcp structure. A snapshot of the cross-section of a simulated critical nucleus is shown in Fig. 3. From a direct inspection of the nuclei we found that the structure of the nuclei is rhcp. In order to be able to carry out the stacking analysis the nuclei needed to have a size of at least 150 particles, otherwise the number of layers is too small to distinguish in a meaningful way between different stackings. To study the structure of even smaller nuclei we performed a local bond-order analysis. We set up a set of vectors, $\mathbf{v}_{\text {rhcp }}, \mathbf{v}_{\text {bcc }}, \mathbf{v}_{\text {ico }} \mathbf{v}_{\text {liq }}$, which contain the characteristic distribution functions of the bond-order parameters, $q_{4}, q_{6}, \hat{w}_{4}, \hat{w}_{6}$, of the relevant lattice structures, i.e. rhcp, bcc, ico and the liquid structure, see also Fig 4. In our simulation the distribution functions for the cluster were also calculated. The corresponding vector is $\mathbf{v}_{\mathrm{cl}}$. The vector $\mathbf{v}_{c l}$ was then decomposed by minimizing the difference $\Delta$ : 


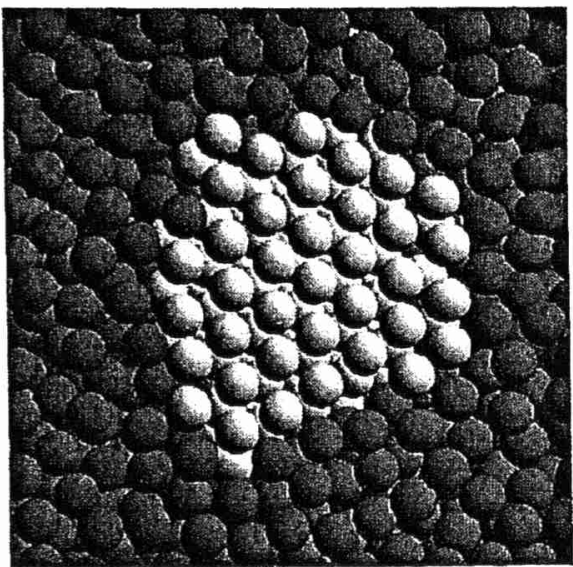

Fig. 12. Snapshot of a cross-section of a critical nucleus of a hard-sphere crystal at a liquid volume fraction $\phi=0.5207$. The figure shows a three-layer thick slice through the center of the crystallite. Solid-like particles are shown in yellow and liquid-like particles in blue. The layers shown in the figure are close-packed hexagonal crystal planes. The stacking shown in this figure happens to be fcc-like, i.e. ABC-stacking - however, analysis of many such snapshots showed that fcc and hcp stackings were equally likely

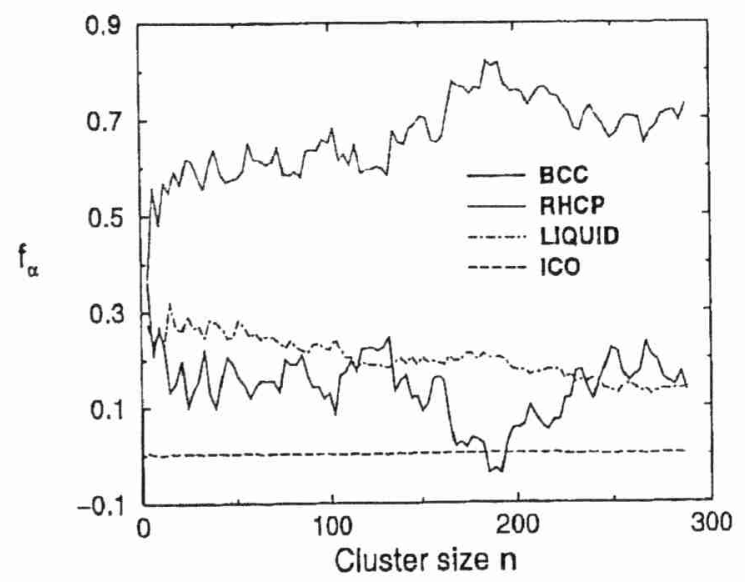

Fig. 13. Structure analysis of (pre) critical crystal nuclei. The figure shows the relative weight of the structural signatures for rhcp, bcc, icosahedral and liquidlike ordering in hard-sphere crystal nuclei of size $n$ 


$$
\Delta=\left\{\mathbf{v}_{\mathrm{cl}}-\left(f_{\text {rhcp }} \mathbf{v}_{\text {rhcp }}+f_{\text {bcc }} \mathbf{v}_{\text {bcc }}+f_{\text {ico }} \mathbf{v}_{\text {ico }}+f_{\text {liq }} \mathbf{v}_{\text {liq }}\right)\right\}^{2} .
$$

The coefficients $f_{\text {rhcp }}, f_{\text {bcc }}, f_{\text {ico }}, f_{\text {liq }}$ are a measure for the type of order in the system. If we apply this analysis to an equilibrated bcc crystal, we would get $f_{\mathrm{bcc}}=1$ and zero for the others. In Fig. 13 we show the results for $f_{\text {rhcp }}, f_{\text {bcc }}, f_{\text {ico }}$ and $f_{\text {liq }}$ as a function of the size of the largest cluster in the system at $P=15$. The results for $P=16$ are qualitatively similar. The figure shows that $b c c$ and icosahedral structures play no role in the nucleation process. Small clusters are fairly disordered and have an appreciable liquidlike signature. The figure shows that the rhcp signature is dominant for all cluster sizes. This was also found in more recent simulations by O'Malley and Snook [50]. Surprisingly, these simulations also found evidence for multiply twinned nuclei with a decahedral morphology.

\section{4}

\section{Effect of Polydispersity}

In practice, the colloidal particles used in the experiments have a distribution of particle radii (referred to as polydispersity) that is rarely less than $2-3 \%$ of the average radius. In order to compare our measured nucleation rates with experiments we already needed to study the effect of a small polydispersity (up to 5\%) in the preceding section. For polydispersities up to $5 \%$, we found no effect of polydispersity on the height of the nucleation barrier. However, experiments on hard-sphere colloids indicate that crystallization is suppressed if the polydispersity exceeds $12 \%$ [51]. This suppression of crystallization is usually attributed to the fact that in poly-disperse suspensions the freezing point is shifted to higher densities where the system tend to become glassy. In a glass, the kinetic pre-factor $\Gamma$ is expected to be very small, but the nucleation barrier itself should continue to decrease with increasing supersaturation. To test this, we studied the behavior of the crystal-nucleation barrier for polydispersities up to $10 \%$ [35].

We performed Monte Carlo in the constant-pressure, semi-grand-canonical ensemble of the type described in [52]. In such a simulation it is not possible to impose the size distribution of the particles directly, but the size distribution can be controlled through variation of the imposed activity-ratio distribution function. In our simulations we imposed a Gaussian activity distribution and a typical particle size distribution function is shown in Fig. 14.

In these simulations, we computed the crystal-nucleation barrier and the structure of the critical nucleus, as a function of both polydispersity and supersaturation. As in the case of monodisperse suspensions [32], we find that all critical nuclei have a randomly-stacked close-packed structure. During crystallization, size-fractionation occurs $[52,53]$ : the particles that make up the critical nucleus are on average larger, and the polydispersity is lower, than those in the metastable liquid, as is shown in Figs. 14 and 15.

We find that $\Delta G^{*}$, the height of the nucleation barrier, at fixed $|\Delta \mu|$, does not depend on the polydispersity for polydispersities $\leq 5 \%$ (see Fig. 16). However, as 


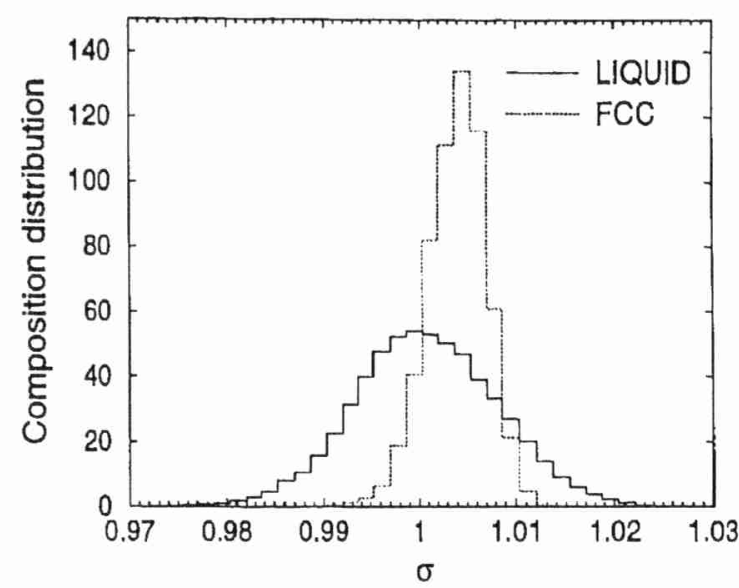

Fig. 14. Typical particle size distribution functions from Monte Carlo simulations in the constant-pressure, semi-grand-canonical ensemble in a bulk liquid and solid. At that pressure the volume fraction of the liquid is $\phi=0.5775$ at a polydispersity of $10 \%$. The volume fraction of the solid in coexistence with the liquid is $\phi=0.6196$ and has a polydispersity of $4.2 \%$

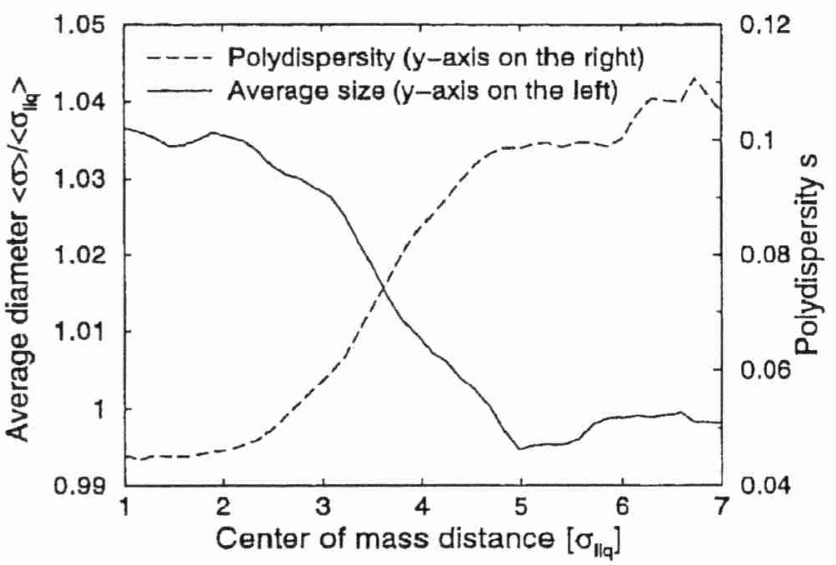

Fig. 15. Average size and polydispersity of particles as a function of the center of mass of the cluster in units of the average diameter of particles in the bulk liquid. Here the average size is scaled to the diameter of the particles in the liquid $\langle\sigma\rangle /\left\langle\sigma_{l i q}\right\rangle$. The polydispersity is defined as $s^{2}=\left\langle\sigma^{2}\right\rangle /\langle\sigma\rangle-1$. Fractionation in particle size and polydispersity occurs. The particles in the crystal nucleus are in average larger as the particles in the bulk liquid, while they are less polydisperse 


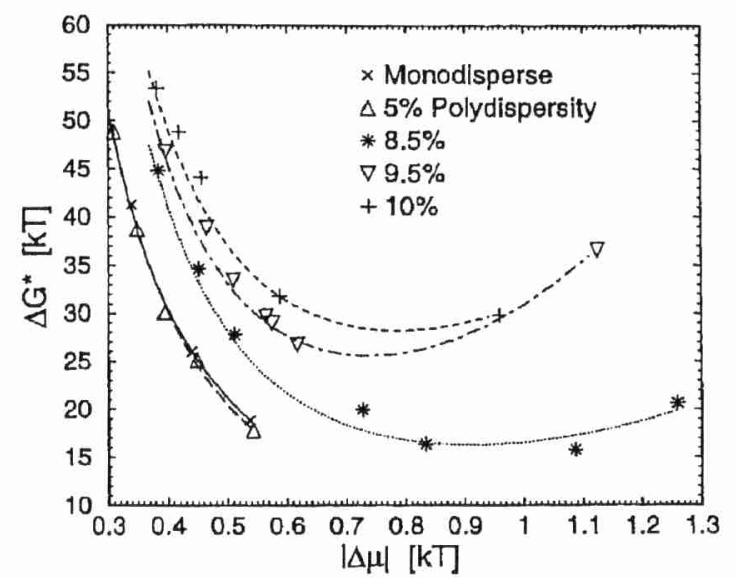

Fig. 16. Computed dependence of the free-energy barrier $\Delta G^{*}$ for crystal nucleation of polydisperse suspensions of hard, colloidal spheres. The free energy is expressed in terms of $k_{B} T$, where $k_{B}$ is Boltzmann's constant and $T$ is the absolute temperature. $|\Delta \mu|$ (also in units of $k_{B} T$ ) is the absolute difference between the chemical potential of the liquid and the solid. It is a measure for the degree of supersaturation. The curves are fits that have been drawn as a guide to the eye. To facilitate comparison with experiment, we have collected in Table 2, the relation between $|\Delta \mu|$ and the volume fraction $\phi$ of the liquid, for the different systems that we studied

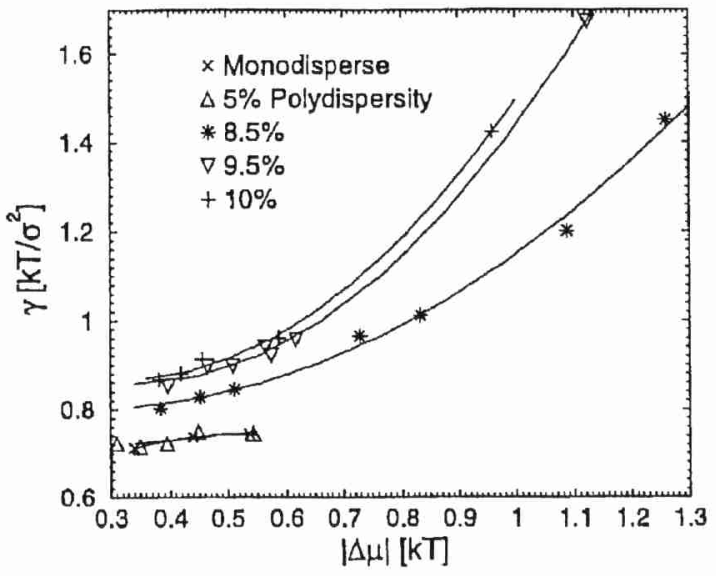

Fig. 17. Dependence of the interfacial free energy $\gamma$ of crystal nuclei in polydisperse suspensions of hard, colloidal spheres. The interfacial free energy is expressed in terms of $k_{B} T / \sigma^{2}$, where $\sigma$ is the average hard-sphere diameter. The curves are fits that have been drawn as a guide to the eye 
the polydispersity is increased beyond $5 \%, \Delta G^{*}$ increases rapidly. This implies that the probability to form a critical nucleus, is suppressed in polydisperse suspensions.

It follows from Eq. (2) (or actually from its polydisperse equivalent, see Appendix $\mathrm{C}$ ), that at constant $|\Delta \mu|$, the variation of $\Delta G^{*}$ with polydispersity is due to an increase of the interfacial free energy $\gamma$, see Fig. 17.

Table 2. Supersaturation and volume fraction of polydisperse colloids. $\Delta \mu$ is the supersaturation and $\phi$ is the volume fraction of the colloidal fluid. For a calculation of $\Delta \mu$ in polydisperse systems see Ref. [8]. The polydispersity ranges from $0 \%$ (left) to $10 \%$ (right). The polydispersities quoted in this table and in the figures, are those of the metastable liquid

\begin{tabular}{cc|cc|cc|cc|cc}
\hline \multicolumn{2}{c|}{$0 \%$} & \multicolumn{2}{c|}{$5 \%$} & \multicolumn{2}{c|}{$8.5 \%$} & \multicolumn{2}{c|}{$9.5 \%$} & \multicolumn{2}{c}{$10 \%$} \\
\hline$\Delta \mu$ & $\phi$ & $\Delta \mu$ & $\phi$ & $\Delta \mu$ & $\phi$ & $\Delta \mu$ & $\phi$ & $\Delta \mu$ & $\phi$ \\
0.339 & 0.5207 & 0.310 & 0.5344 & 0.385 & 0.5614 & 0.397 & 0.5697 & 0.382 & 0.5717 \\
0.439 & 0.5277 & 0.349 & 0.5377 & 0.451 & 0.5673 & 0.465 & 0.5746 & 0.419 & 0.5738 \\
0.538 & 0.5342 & 0.395 & 0.5414 & 0.512 & 0.5726 & 0.509 & 0.5782 & 0.455 & 0.5775 \\
& & 0.448 & 0.5456 & 0.728 & 0.5864 & 0.565 & 0.5808 & 0.587 & 0.5878 \\
& & 0.544 & 0.5528 & 0.833 & 0.5948 & 0.575 & 0.5828 & 0.959 & 0.6239 \\
& & & 1.088 & 0.6145 & 0.616 & 0.5859 & & \\
& & & 1.260 & 0.6212 & 1.125 & 0.6239 & & \\
\hline
\end{tabular}

The increase of $\gamma$ with polydispersity runs counter to Turnbull's suggestion that the interfacial free energy should be proportional to $\Delta H$, the latent heat of fusion [3]. For the systems that we studied, $\Delta H$ crosses zero at a polydispersity of $9 \%$ [54], where the liquid becomes denser than the coexisting solid [52]. Yet, $\gamma$ clearly remains non-zero, see Fig. 18.

Surprisingly, the variation of $\Delta G^{*}$ with $|\Delta \mu|$ is non-monotonic. As $|\Delta \mu|$ is increased, the nucleation barrier goes through a minimum (Fig. 16). This nonmonotonic behavior of $\Delta G^{*}$ is due to the increase of $\gamma$ with $|\Delta \mu|$. To illustrate this, let us approximate the $|\Delta \mu|$-dependence of $\gamma$ by $\gamma \approx \gamma_{0}(1+a|\Delta \mu|)$. Ignoring the slight $|\Delta \mu|$-dependence of the solid density, it then follows from Eq. (2) that $\Delta G^{*}$ must go through a minimum when $|\Delta \mu|=2 / a$. The nucleation theorem $[55,56,57]$ suggests that the minimum in $\Delta G^{*}$ is due to the inversion of the densities of the polydisperse fluid and the crystal nucleus. In CNT it is usually assumed that $\gamma$ is constant. A linear variation of $\gamma$ with $|\Delta \mu|$ has been observed in inorganic glasses [3], but there the constant $a$ is negative and hence there is no minimum in $\Delta G^{*}$. In other systems $[58,59]$, non-monotonic behavior of $\Delta G^{*}$ is induced by a hidden phase transition in the meta-stable phase.

The minimum value of $\Delta G^{*}$ increases rapidly with polydispersity. Using kinetic Monte Carlo simulations, we can estimate the value of the attachment rate [19]. We find that, over the range of supersaturations studied, the attachment rates vary by at most an order of magnitude (Table 3). This means that the variation in the rate of nucleation is dominated by the behavior of $\Delta G^{*}$. We estimate that, for colloidal particles with a radius $\geq 500 \mathrm{~nm}$, homogeneous nucleation will be effectively sup- 


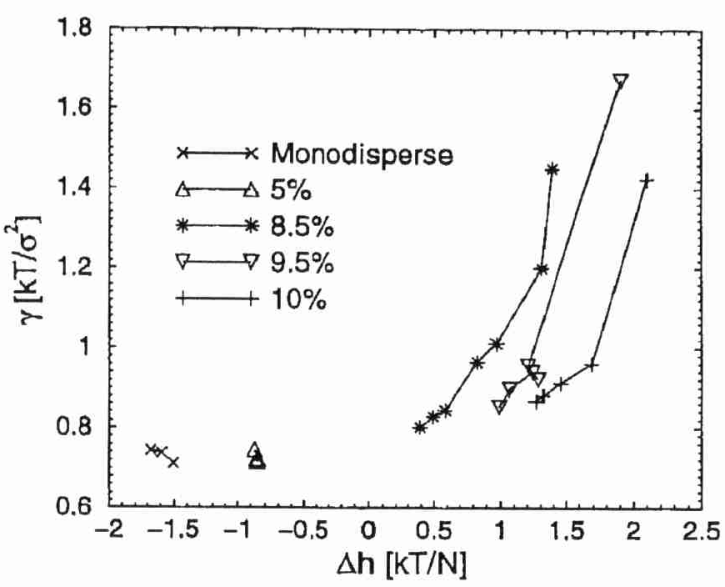

Fig. 18. Surface free energy $\gamma$ as a function of the enthalpy difference $\Delta h$ (per particle) between the liquid and the crystal phase shown for the different polydispersities

pressed (less than one nucleus per $\mathrm{cm}^{3}$ per day) when the polydispersity exceeds $10 \%$. This finding has important implications for the morphology of polycrystalline colloidal materials. Using a simplified version of the analysis proposed by Shi et al.

Table 3. Reduced attachment rate $f_{n_{c}}^{+} / D_{0}$ as a function of the volume fraction $\phi$

\begin{tabular}{cc|cc|cc}
\hline \multicolumn{2}{c|}{$8.5 \%$} & \multicolumn{2}{|c|}{$9.5 \%$} & \multicolumn{2}{c}{$10 \%$} \\
\hline$f_{n_{c}}^{+} / D_{0}$ & $\phi$ & $f_{n_{c}}^{+} / D_{0}$ & $\phi$ & $f_{n_{c}}^{+} / D_{0}$ & $\phi$ \\
40 & 0.5614 & 12 & 0.5697 & 20 & 0.5717 \\
75 & 0.5673 & 60 & 0.5746 & 55 & 0.5738 \\
21 & 0.5726 & 15 & 0.5782 & 40 & 0.5775 \\
30 & 0.5864 & 50 & 0.5808 & 10 & 0.5878 \\
15 & 0.5948 & 10 & 0.5828 & 8 & 0.6239 \\
35 & 0.6145 & 20 & 0.5859 & & \\
10 & 0.6212 & 5 & 0.6239 & & \\
\hline
\end{tabular}

[58] we can estimate the size of crystallites in a polycrystalline sample at the end of a crystallization experiment. We assume that $I$, the rate of steady-state nucleation, is given by Eq. (4), and that $v_{g}$, the rate at which the crystallite radius grows, is given by the Wilson-Frenkel law:

$$
v_{g}=\frac{D_{S}}{\lambda}\left[1-\exp \left(-|\Delta \mu| / k_{B} T\right)\right],
$$

where $\lambda$ is a typical atomic jump distance and $D_{S}$ a self-diffusion constant. Note that both $I$ and $v_{g}$ are proportional to $D_{S}$. The total volume fraction occupied by crystallites as a function of time $t$ is approximately given by the Avrami growth law 


$$
\phi \approx I \pi v_{g} t^{4} / 3 .
$$

Crystallization stops when $\phi$ is of order 1 . This happens after a time $t_{\max } \sim$ $\left(I v_{g}^{3}\right)^{-1 / 4}$. The average crystallite radius at this time is equal to $R_{c} \approx v_{g} t_{\max }$. Using the expression for $t_{\max }$ above, it follows that $R_{c} \sim\left(v_{g} / I\right)^{1 / 4}$. The crucial point to note is that the average crystallite size depends only on the ratio $v_{g} / I$. As the strongly density dependent diffusion constant $D_{S}$ drops out of this ratio, its $|\Delta \mu|$-dependence is mainly determined by the variation of $\exp \left(\Delta G^{*} / k_{B} T\right)$, except for small supersaturations. We therefore expect that the typical crystallite size at the end of a nucleation experiment should scale as $R_{c} \sim \exp \left(\Delta G^{*} / 4 k_{B} T\right)$. This should be experimentally observable.

We could only compute $\Delta G^{*}$ if spontaneous nucleation did not occur in the course of a simulation. In practise, this implied that we could not study barriers lower than $15 k_{B} T$. As a result, we could not test whether $\Delta G^{*}$ in systems with a low polydispersity (less than $8.5 \%$ ) also has a minimum. If we assume that, also at lower polydispersities, we can extrapolate the increase of $\gamma$ with $|\Delta \mu|$ to large supersaturations, then we predict that a minimum in $\Delta G^{*}$ should occur even in nearly monodisperse systems. Again, this should be experimentally observable, because we should expect to see the formation of larger crystallites if the solution can be compressed rapidly through the region where $\Delta G^{*}$ is small.

There are two ways to interpret the experimental finding that crystallization is not observed in suspensions with a polydispersity $>12 \%$ : either crystals do not form, or they are too small to be observed. Our simulations support the first interpretation. Using Shi's approach, we can estimate the maximum number of crystallites per unit volume [58]. For a suspension of colloids with a 500 -nm radius, we expect to see less than one crystallite per cubic centimeter, once $\Delta G^{*}>32 k_{B} T$. In other words, under those conditions the colloidal glass is truly amorphous.

Our predictions concerning the structure and free energy of colloidal crystal nuclei can be tested experimentally. Recently, the technique of confocal scanning laser microscopy has been applied by Gasser et al. [60] to study the structure and size of critical crystal nuclei in dense colloidal suspensions. This technique would be perfectly suited to test our predictions. Our prediction concerning the minimum in $\Delta G^{*}$ is even easier to verify. By visual inspection, one could verify whether the crystallites that nucleate in strongly supersaturated solutions are larger than those that form at lower supersaturations. Over a decade ago, Pusey and van Megen published beautiful images of the morphology of poly-crystalline hard-sphere colloids [29] (similar morphologies have recently been observed in a study of colloidal crystallization in micro-gravity - Z.D. Cheng, W.B. Russel and P.M. Chaikin, unpublished data). Pusey and van Megen observed an increase of the crystallite size at large supersaturations. However, they attributed this effect to heterogeneous nucleation. Hence, a direct test of our prediction is still lacking. 


\section{5}

\section{Weakly Charged Colloids}

Experiments on colloidal crystallization consistently show that it is much easier to crystallize charged colloids than uncharged ("hard-sphere") colloids. Clearly, longranged repulsion has a large effect on the crystal-nucleation rate. This may even be true for colloidal suspensions of particles that are only weakly charged. Using simulations, it is possible to study in detail how repulsive inter-particle forces affect the crystal-nucleation process [61]. The aim of this section is to elucidate the factors that affect the rate of crystal nucleation in a system of weakly charged colloids.

In suspension, the charged colloids are surrounded by a cloud of counterions. This counterion double layer screens the pure Coulomb interaction between the colloids. If we use the linearized Poisson-Boltzmann equation to describe the charge distribution around a charged colloid with hard-core diameter $\sigma$, then we obtain the following expression for the pair interaction between two charged macro-ions:

$$
\beta U(r)= \begin{cases}\infty & \text { for } r<\sigma \\ \beta \epsilon \frac{\exp (-\kappa(r / \sigma-1))}{r / \sigma} & \text { for } r>\sigma .\end{cases}
$$

$U(r)$ is usually referred to as the "hard-core Yukawa potential". Here $\kappa$ is the inverse screening length in units of the hard-sphere diameter $\sigma$ and $\beta \epsilon$ is the value of the Yukawa repulsion at contact. $\beta$ is a measure for the inverse temperature $\left(\beta=1 / k_{B} T\right)$, where $k_{B}$ is the Boltzmann constant. In the linearized PoissonBoltzmann theory, we have explicit expressions for both $\kappa$ and $\epsilon$ in terms of the size and surface charge of the colloid, and of the concentration of counterions and added salt. However, the linearized Poisson-Boltzmann description provides only an approximation to the real colloid-colloid interaction. For instance, it is expected to break down at short distances and for low added salt concentrations. A way to treat the interaction between charged colloids at short distances was already proposed by Derjaguin, Landau, Verweij and Overbeek (DLVO) in the 1940s [62]. Since then, several modifications of the form of the pair potential between charged colloids have been proposed $[63,64]$ but, except at very short distances, most expression are very similar to the hard-core Yukawa model. The main difference between the theories is the values that they yield for $\kappa$ and $\epsilon$. In the original DLVO theory, these parameters depend only on the ionic strength of the solution and on the bare charge of the colloids. In the more recent theories, $\kappa$ and $\epsilon$ may themselves depend on the concentration of charged colloids. In the present work, we simply assume that the interaction between charged colloids is adequately described by a hard-core Yukawa potential. However, we shall return later to the question whether this is allowed. A special case of the hard-core Yukawa model, is the hard-sphere model. The latter model applies in the limit of high salt concentrations $\kappa \rightarrow \infty$ and in the limit that the strength of the repulsion is much less than the thermal energy, i.e. $\beta \epsilon \rightarrow 0$. This is typically the case for weakly charged colloids. We note that, whilst the hard-core Yukawa model is commonly used to describe slightly charged colloids, it can also be used as a crude model for sterically stabilized colloids. Hence, many of the con- 
clusions that we obtain below, in particular those for systems with a high value of $\kappa$, should equally apply to sterically stabilized, uncharged colloids.

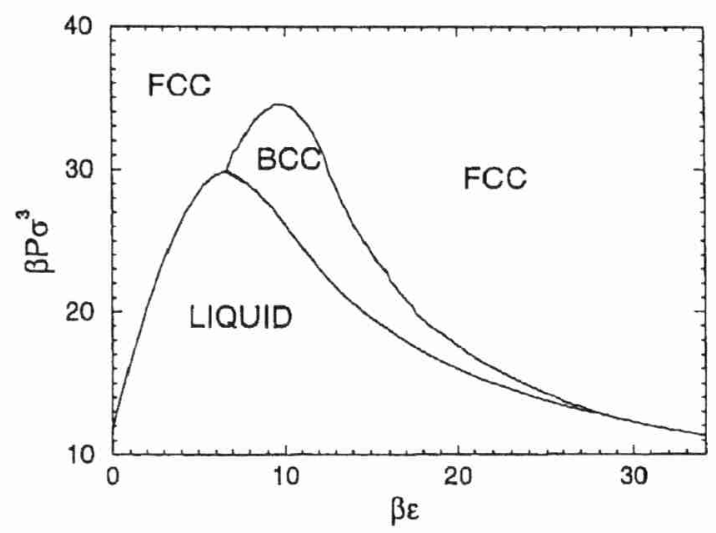

Fig. 19. Calculated coexistence pressure from Ref. [67] for $\kappa=5$ as function of the Yukawa repulsion $\beta \epsilon$

The phase behavior of the hard-core Yukawa potential has been studied experimentally and by numerical simulation, see e.g. Ref. $[65,66,67]$. The computed phase diagram of Ref. [67] shows a fluid-solid (bcc/fcc) and a solid-solid (bcc-fcc) coexistence line and it exhibits two fluid-bcc-fec triple points, (see Fig.19). The main difference between the phase diagram of the hard-core Yukawa model and that of the pure (i.e. point-particle) Yukawa potential [68] is the presence of the second triple point. This triple point sets a lower limit for the strength of the Yukawa interaction for which a bcc phase exists.

Only few nucleation experiments on charged colloidal suspensions have been reported. Some of these were based on light-scattering studies [69, 70]. More recently, Gasser et al. [60] reported a confocal microscopy study of homogeneous crystal nucleation in slightly charged hard-sphere colloids. Recent light-scattering experiments of crystallization in more highly charged colloids has been reported by Schöpe et al. [71] and Wette [72].

We performed a computer-simulation study of crystal nucleation in a hard-core Yukawa system varying both parameters, the amplitude of the Yukawa repulsion and the magnitude of screening length. First we computed the nucleation barrier at fixed $\kappa=5$ for four different values of the amplitude of the Yukawa repulsion $\beta \epsilon=2,6,8$ and 20 . Increasing the contact value $\beta \epsilon$ of the Yukawa repulsion shifts the volume fraction of the liquid phase at freezing to lower values than the hardsphere value $\phi=0.494$. In order to be able to interpret our numerical data on the free-energy barrier for crystal nucleation, we need an accurate estimate of the density, pressure and chemical potential of the liquid at freezing. The data of Ref. [67] were obtained using a (modified) Gibbs-Duhem integration method. While this tech- 
Table 4. Excess free energy per particle for the different bulk structures and the liquid state calculated via a thermodynamic intergration in the limit of infinite number of particles [102]. The reference state for the free energy calculation of the liquid was the hard-sphere fluid, and for the bulk solid structures we used an Einstein-crystal. In some cases we also used the hardsphere system as a reference state for the solid structures. We found that the solid free energies obtained via these two distinct routes agreed to within $\pm 0.005 k_{B} T$, which corresponds to our estimate of the statistical error in this calculation. The statistical accuracy of the computed free energy of the liquid is estimated to be $\pm 0.01 k_{B} T$. In the table, the values in brackets indicate the volume fraction at which the excess free energy was calculated. The calculated excess free energies for the fcc and the hcp structures can be compared directly, as they were calculated at the same pressure, whereas the others are not. The fcc-hcp free energy difference is always smaller than $\left(1 \times 10^{-2} k_{B} T\right)$

\begin{tabular}{ccccc}
\hline & $f_{\text {fcc }}$ & $f_{\text {hcp }}$ & $f_{\text {bcc }}$ & $f_{\text {liquid }}$ \\
\hline$\beta \epsilon=2$ & 12.894 & 12.892 & - & 11.38 \\
$\kappa=5$ & $(0.5425)$ & $(0.5425)$ & - & $(0.5032)$ \\
\hline$\beta \epsilon=6$ & 23.258 & 23.256 & 21.49 & 19.11 \\
$\kappa=5$ & $(0.5027)$ & $(0.5027)$ & $(0.4808)$ & $(0.4503)$ \\
\hline$\beta \epsilon=8$ & 24.344 & 24.35 & 24.32 & 22.23 \\
$\kappa=5$ & $(0.4563)$ & $(0.4563)$ & $(0.4558)$ & $(0.4329)$ \\
\hline$\beta \epsilon=20$ & 20.872 & 20.873 & 20.986 & 16.16 \\
$\kappa=5$ & $(0.2888)$ & $(0.2888)$ & $(0.2895)$ & $(0.2529)$ \\
\hline$\beta \epsilon=8$ & 11.144 & 11.147 & 11.067 & 10.02 \\
$\kappa=10$ & $(0.4084)$ & $(0.4084)$ & $(0.4054)$ & $(0.3853)$ \\
\hline$\beta \epsilon=8$ & 39.107 & 39.110 & - & 38.08 \\
$\kappa=3.33333$ & $(0.5168)$ & $(0.5168)$ & - & $(0.5055)$ \\
\hline
\end{tabular}

nique is useful to estimate the location of solid-liquid coexistence curves, the computed coexistence data were not sufficiently accurate for the present purpose. We therefore computed the location of all coexistence points by direct free-energy calculation of the solid and liquid phases [15]. The results for the excess free energy per particle are summarized in Table 4 . From the computed free energies, we obtain estimates for the chemical potential at freezing that have an error of $\pm 0.01 k_{B} T$. We found the following values for the volume fraction of the liquid phase at freezing: $\phi=0.48212,0.43823,0.4049$ and 0.26171 for $\beta \epsilon=2,6,8$ and 20 , respectively (see Table 5). In Fig. 20 we show the results for the barrier height as a function of supersaturation with respect to the stable solid phase (fcc). As the figure shows, the main effect of increasing the strength of the Yukawa repulsion is to lower the nucleation barrier at constant supersaturation $\Delta \mu$.

Note that the decrease of the height of the nucleation barrier is particularly strong when only a weak repulsion is added to the hard-core potential. In particular, switching on a repulsive Yukawa potential with a contact value of only $2 k_{B} T$ decreases the nucleation barrier by some $10 k_{B} T$. This implies that for real hard-sphere colloids, 


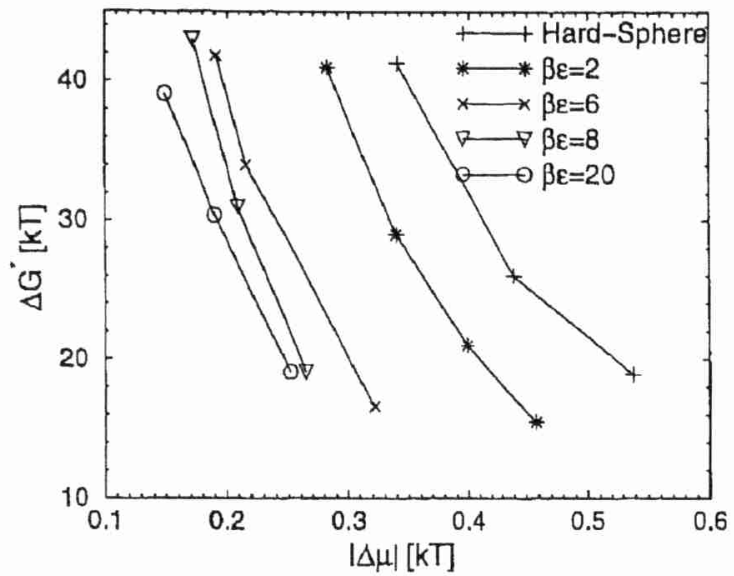

Fig. 20. Calculated barrier heights $\Delta G^{*}$ of the hard-core Yukawa system with $\kappa=5$ and $\beta \epsilon=2,6,8,20$ plotted as a function of supersaturation $\Delta \mu$ of the liquid phase to the stable fcc phase

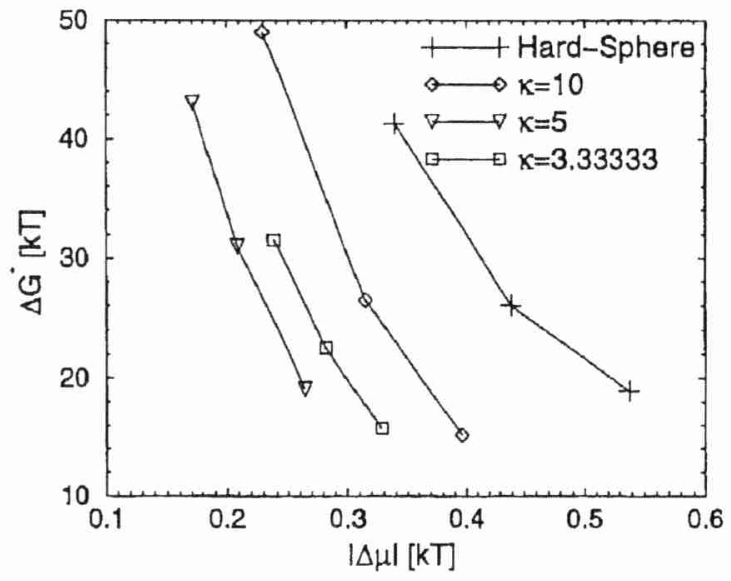

Fig. 21. Calculated barrier heights $\Delta G^{*}$ of the hard-core Yukawa system with $\beta \epsilon=8$ and $\kappa=10,5,3.33333$ plotted as a function of supersaturation $\Delta \mu$ of the liquid phase to the stable fcc phase 


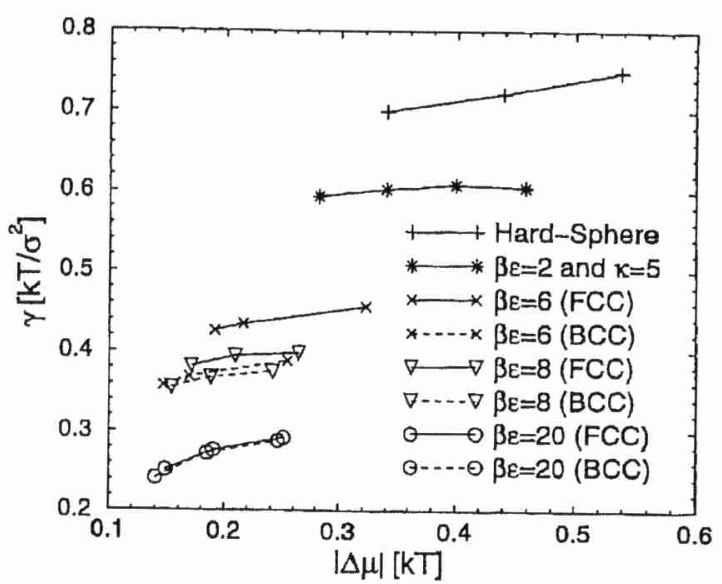

Fig. 22. Interfacial free energy $\gamma$ calculated from the barrier heights Eq. 2 for $\kappa=5$ and $\beta \epsilon=2,6,8,20$. The solid lines are the results assuming that the nuclei have a fcc structure, and the dashed lines are the results if the nuclei are bcc

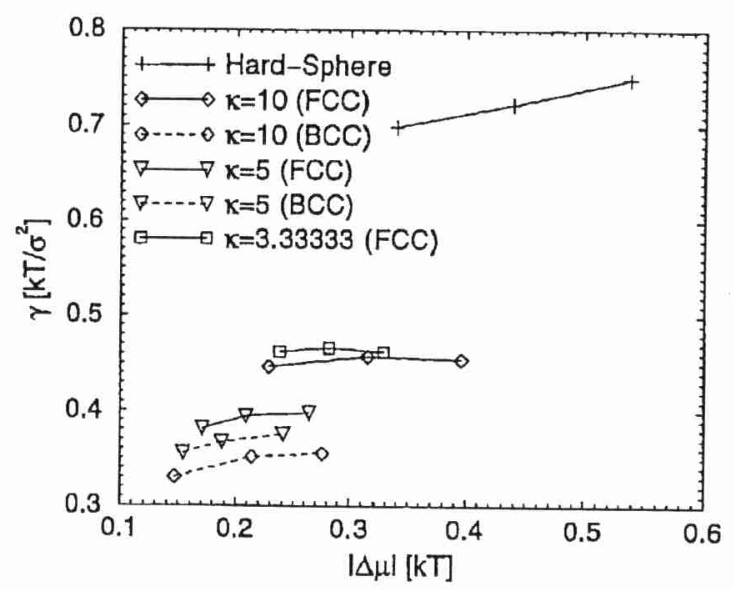

Fig. 23. Interfacial free energy $y$ calculated from the barrier heights Eq. 2 for $\beta \epsilon=8$ and $\kappa=10,5,3.33333$. The solid lines are the results assuming that the nuclei have a fcc structure, and the dashed lines are the results if the nuclei are bcc

the presence of only a small amount of charge can enhance the nucleation rate at constant volume fraction by many orders of magnitude through two mechanisms: first of all, the charge increases the supersaturation at constant density. This effect would shift the nucleation curve to lower densities. But, in addition, the charge lowers the nucleation barrier at constant supersaturation. Further increase of the strength of the Yukawa repulsion leads to some additional decrease of the nucleation barrier, but the effect seems to saturate for values of $\beta \epsilon$ between 8-20. 
Table 5. Summary of the data for the calculations with the repulsive hard-core Yukawa potential. Here $P$ is the Pressure and $\phi_{\mathrm{liq}}, \phi_{\mathrm{fcc}}, \phi_{\mathrm{bcc}}$ the corresponding volume fraction of the liquid, fcc and bcc phase. $\Delta \mu_{\mathrm{fcc}}$ and $\Delta \mu_{\mathrm{bcc}}$ is the difference in chemical potential between the liquid and the fcc/bcc phases. $\Delta G^{*}$ are the measured crystallization barriers. $f_{n_{\mathrm{c}}}^{+} / D_{0}$ is the reduced attachment rate of particles to the critical cluster. I is the calculated reduced nucleation rate

\begin{tabular}{cccccccccc}
\hline & $P$ & $\phi_{\text {liq }}$ & $\phi_{\mathrm{fcc}}$ & $\phi_{\mathrm{bcc}}$ & $\Delta \mu_{\mathrm{fcc}}$ & $\Delta \mu_{\mathrm{bcc}}$ & $\Delta G^{*}$ & $f_{n_{c}}^{+} / D_{0}$ & $\log _{10}(I)$ \\
\hline$\kappa=5$ & 25 & 0.5103 & 0.5420 & - & 0.28 & - & 41 & 46 & -19.1 \\
and & 26 & 0.5159 & 0.5484 & - & 0.34 & - & 29 & 84 & -13.5 \\
$\beta \epsilon=2$ & 27 & 0.5218 & 0.5551 & - & 0.40 & - & 21 & 6 & -11.1 \\
& 28 & 0.5257 & 0.5599 & - & 0.46 & - & 15.5 & 19 & -8.1 \\
\hline$\kappa=5$ & 37 & 0.4714 & 0.4827 & 0.4808 & 0.19 & 0.15 & 48.1 & 202 & -19 \\
and & 38 & 0.4755 & 0.4864 & 0.4848 & 0.22 & 0.17 & 34 & 57 & -16.1 \\
$\beta \epsilon=6$ & 42 & 0.4903 & 0.5031 & 0.5004 & 0.32 & 0.25 & 16.6 & 52 & -8.3 \\
\hline$\kappa=5$ & 38 & 0.4415 & 0.4487 & 0.4481 & 0.17 & 0.15 & 43 & 218 & -19.5 \\
and & 40 & 0.4491 & 0.4563 & 0.4558 & 0.21 & 0.19 & 31 & 200 & -14.3 \\
$\beta \epsilon=8$ & 43 & 0.4596 & 0.4671 & 0.4668 & 0.26 & 0.24 & 19.1 & 300 & -8.8 \\
\hline$\kappa=5$ & 23 & 0.2859 & 0.2888 & 0.2895 & 0.15 & 0.14 & 39.1 & 167 & -18.2 \\
and & 25 & 0.2938 & 0.2973 & 0.2974 & 0.19 & 0.19 & 30.4 & 58 & -14.8 \\
$\beta \epsilon=20$ & 28 & 0.3048 & 0.3084 & 0.3083 & 0.25 & 0.25 & 19.1 & 53 & -9.7 \\
\hline$\kappa=10$ & 18 & 0.3848 & 0.3978 & 0.3949 & 0.23 & 0.15 & 49 & 80 & -22.6 \\
and & 20 & 0.3955 & 0.4084 & 0.4054 & 0.32 & 0.21 & 26.5 & 44 & -13 \\
$\beta \epsilon=8$ & 22 & 0.4054 & 0.4180 & 0.4150 & 0.40 & 0.28 & 15.2 & 11 & -8.5 \\
\hline$\kappa=3.33333$ & 57 & 0.4937 & 0.5042 & - & 0.24 & - & 31.5 & 205 & -14.4 \\
and & 59 & 0.4996 & 0.5106 & - & 0.28 & - & 22.5 & 81 & -10.8 \\
$\beta \epsilon=8$ & 61 & 0.5055 & 0.5168 & - & 0.33 & - & 15.8 & 80 & -7.7 \\
\hline
\end{tabular}

Let us next consider the effect of the range of the repulsive potential on the nucleation barrier. We computed the height of the crystallization barrier for $\kappa=10$, 5 and 3.333 at a fixed contact value $\beta \epsilon=8$. In addition, we know the behavior of the system in the hard-sphere limit $(\kappa=\infty)$. As $\kappa$ is decreased, the range of the potential grows. Initially, (as $\kappa$ is decreased from $\infty$ to 10 , the density at which the liquid freezes shifts from $\phi=0.494$ to $\phi=0.354$. Subsequently, the freezing density increases again. For $\kappa=5$, the volume fraction at freezing is $\phi=0.405$ and for $\kappa=3.333$, the liquid freezes at $\phi=0.456$. The variation of the crystallization barrier with $\kappa$ and $\Delta \mu$ is shown in Fig. 21. The figure shows that increasing the range of the repulsive interaction, at constant supersaturation, initially has the effect to lower the nucleation barrier. However, as $\kappa$ is decreased below 5 , the nucleation barrier starts to increase again.

From the CNT expression for the height of the nucleation barrier Eq. (2), we can estimate the corresponding values for the liquid/fcc interfacial free energy $\gamma_{\mathrm{fcc}}$. In Fig. 22 we show the variation of the interfacial free energy with $\beta \epsilon$ at fixed $\kappa$. Fig- 


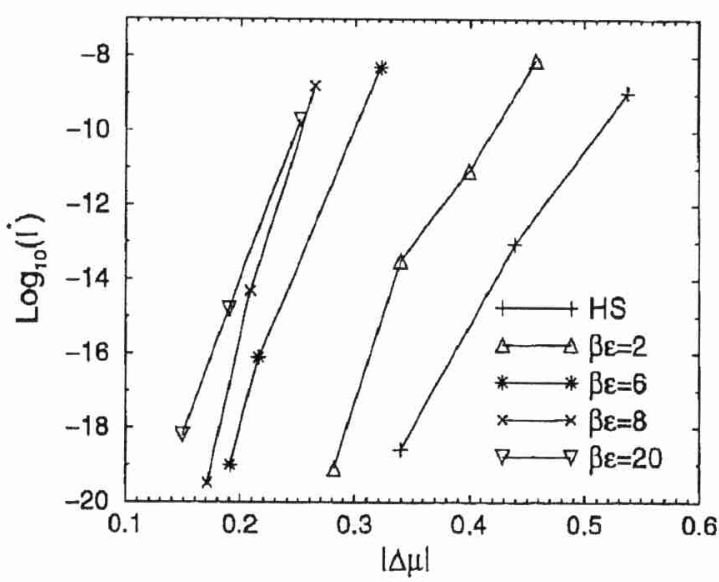

Fig. 24. Dependence of the reduced crystallization rates $I^{*}$ on the amplitude of the Yukawa repulsion $\beta \epsilon=2,6,8,20$ for $\chi=5$ plotted as a function of supersaturation $\Delta \mu$ of the liquid to the stable fcc phase

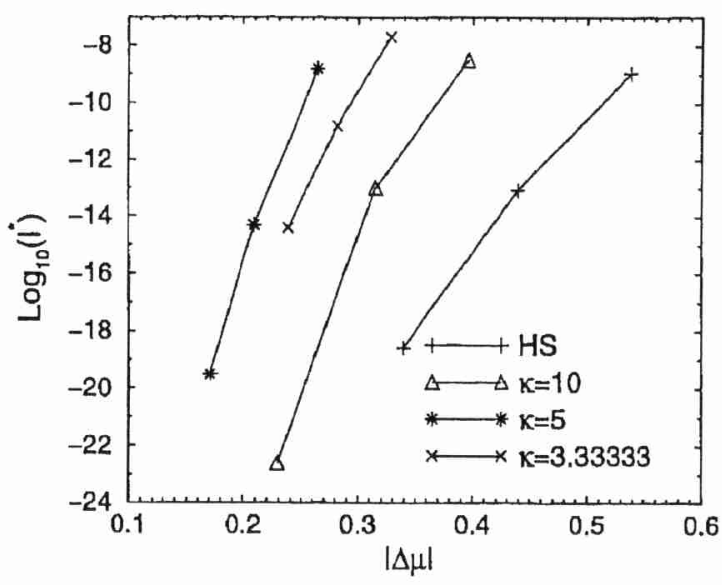

Fig. 25. Dependence of the reduced crystallization rates $I^{*}$ on the inverse screening length $\kappa a=10,5,3.33333$ for $\beta \epsilon=8$ plotted as a function of supersaturation $\Delta \mu$ of the liquid to the stable fcc phase 
ure 23 shows the variation of the interfacial free energy with $\kappa$ at fixed $\beta \epsilon$ for various values of the supersaturation $\Delta \mu$. The dependence of the interfacial free energy on the range of repulsion mirrors that of the nucleation barrier and is therefore nonmonotonic. Coming from the hard-sphere limit, the interfacial free energy initially goes down, but for $\kappa$ less than 5 , it increases again.

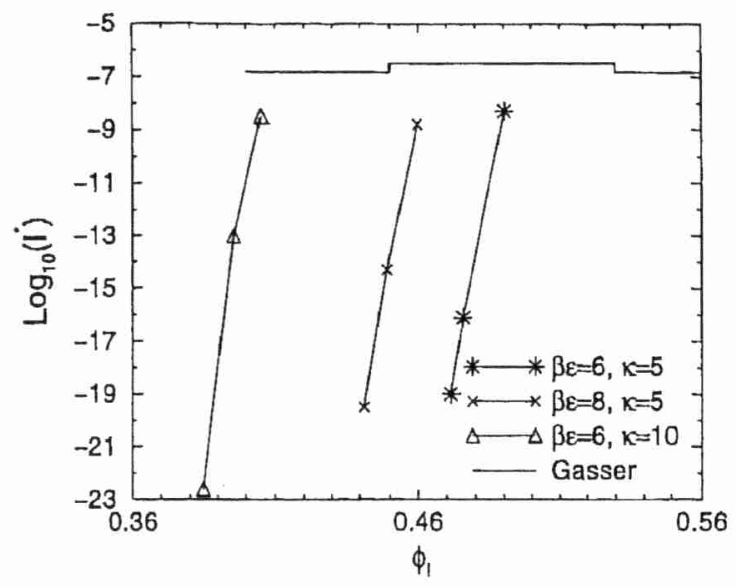

Fig. 26. Comparison between the experimentally measured nucleation rates [60] and the simulation data. The nucleation rates are expressed in reduced units $I^{*}=1 \sigma^{5} / D_{0}$ where $\sigma$ is the hard core diameter and $D_{0}$ is the self-diffusion coefficient at infinite dilution. $\phi_{l}$ is the volume fraction of the liquid. In the plot we added only the data sets that match the freezing volume fraction of the experimental system

In Refs. $[32,35]$ we found that, for hard spheres, the interfacial free energy $\gamma$ increases with supersaturation $\Delta \mu$. As can be seen in Fig. 23, such behavior is also observed in a system of charged colloids. In polydisperse hard-sphere systems [35], the increase of $\gamma$ with supersaturation could even result in a non-monotonic dependence of the nucleation barrier on supersaturation. In the present system, the interfacial free energy also increases with supersaturation, but the effect is not strong enough to result in a minimum in the nucleation barrier.

Using the techniques described before we computed the kinetic prefactors. Note that for the correction of the hydrodynamic interaction we used the expression $D_{S}^{S} / D_{0}=(1-\phi / 0.64)^{1.17}$ for the short time self diffusion coefficient from the hard sphere system. We therefore defined an effective packing fraction of the Yukawa system such that the packing fraction at freezing of the two systems are equal. We find that the kinetic prefactors do not vary strongly with either supersaturation or interaction potential and therefore the behavior of the nucleation rate is reflected by that of the barrier height. Our results for the computed nucleation rates are shown in Figs. 24 and 25, where we plot the nucleation rate as a function of supersaturation. 
It is interesting to compare the computed crystallization rates with the results of the confocal microscopy experiments of Ref. [60]. In order to do this we need to know the potential parameters that best characterize the experimental system used in Ref. [60]. From the fact that the suspensions studied by Gasser et al. freeze at a volume fraction $\phi=0.38$, it is clear that the colloidal particles used in these experiments are slightly charged. It is therefore natural to describe them by a Yukawa model that also has its freezing point at $\phi=0.38$. This condition is, however, not sufficient to fix the values of both $\kappa$ and $\epsilon$. For instance, if $\kappa=5$, then the observed freezing density can be reproduce by choosing $\beta \epsilon \sim 7$. Conversely, if we choose $\beta \epsilon=8$, then there are in fact, two values of $\kappa$ that will reproduce the observed freezing density $(\kappa \sim 20$ and $\kappa \sim 6$ ) [67]. In Fig. 26, we show a comparison of the reduced nucleation rates reported in Ref. [60] with the simulation results for those $\kappa-\beta \epsilon$ combinations that yield a freezing point near $\phi=0.38$. As can be seen from the figure, different $\kappa-\beta \epsilon$ combinations yield very different nucleation rates. However, the main effect of the variation of $\kappa$ and $\epsilon$ is to shift the nucleation curves horizontally: the slopes of the different curves are all rather similar. When we compare the computed nucleation rates with the experimental data, we note two things: first of all, the experimental rates tend to be (much) higher than the computed rates (Gasser et al. find $-6.9 \leq \log \left[I^{*}\right] \leq-6.5$ for $\phi$ between 0.45 and 0.53 ). But, more importantly, the experiments suggest that the nucleation rate barely varies with volume fraction. We were unable to reproduce this behavior with any of the Yukawa models that we studied. This discrepancy between experiment and simulation suggests that the experimental system is not well described by a Yukawa model with density-independent $\kappa$ and $\epsilon$. On the contrary, it is likely that the effective potential parameters of weakly charged colloids in the absence of added salt depend strongly on concentration. In fact, recent experiments by Schöpe et al. [73] clearly illustrate this effect: with increasing concentration, the effective potential of charged polystyrene spheres in dilute aqueous solution, becomes increasingly hard-sphere like. If we assume that the same phenomenon occurs in the more concentrated suspensions of Ref. [60], then experimental results for the nucleation rates at different densities should be compared with the numerical predictions that correspond to different effective Yukawa potentials. As can be seen from Fig. 26, the variation of the nucleation rate with density can be strongly reduced (and can possibly even become non-monotonic) if, as we expect, $\epsilon$ and $\kappa$ decrease with density. It is, however, not obvious that this effect is large enough to account for the apparent discrepancy between experiment and simulation. Clearly, a truly quantitative comparison between simulation and experiment requires better knowledge of the density dependence of the effective interaction between slightly charged colloidal spheres.

The repulsive Yukawa system offers a unique opportunity to study the effect of meta-stable crystal phases on the pathway for crystal nucleation. To study the effect of meta-stable intermediates on crystallization, we analyzed the structure of the (pre)critical nucleus in different regions of the phase diagram. Note that the pressure range region where the bcc phase is stable is rather narrow. For these pressures, the supersaturation of the fluid phase is small, and hence the nucleation barrier is very high. As a consequence, we could only study the formation of pre-critical nuclei in 
the fcc regime. In order to study the structure of the (pre)critical nuclei, we used the local bond-order analysis proposed by ten Wolde et. al. [13]. In this analysis the local bond-order signature of a nucleus is decomposed into the signatures of the different bulk structures (liquid, fcc and bcc) using a linear least square fit. The value of the resulting coefficients $\left\{f_{\text {liq }}, f_{\mathrm{fcc}}, f_{\mathrm{bcc}}\right\}$ are a measure of the structure of the nucleus. Our simulations show that the pre-critical nuclei always have a strong bcc signature. Only for larger (post)critical nuclei well inside the fcc regime, do we find a mixture of bcc and fcc signatures. In this sense, our simulations unambiguously support the prediction that nucleation into bcc nuclei is always uniquely favored, even when the fcc phase is closer in free energy to the fluid phase.

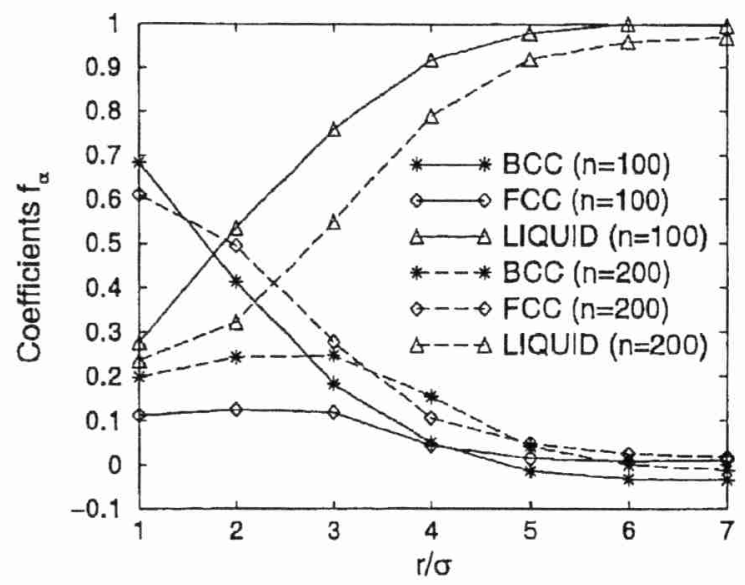

Fig. 27. Structure analysis of two independent crystal nuclei of size $n=100$ and 200 from the simulations with parameters $\beta \epsilon=8$ and $\kappa=10$. The figure shows the results for the fit parameters for the local bond-order analysis as a function of the distance from the center of mass of the nuclei. The core of the cluster of size $n=100$ has a clear bcc signature, where the cluster of size $n=200$ shows a clear fcc structure

Figure 27 shows the results of our cluster analysis for two distinct nuclei of size $n=100$ and $n=200$. The picture shows the variation of the structural signature with the distance from the center of mass of the nucleus. The results shown in this figure apply to the case $\kappa=10$ and $\beta \epsilon=8$. This corresponds to the points in the phase diagram where the preference for the fcc structure is strongest. The core of the cluster of size $n=100$ has a clear bcc signature while the fcc phase does not seem to play a role. However, for the larger nuclei $(n=200)$ the core of the nuclei becomes fcc like while the bcc phase seem to disappear. In this case the cluster transformation happened before it could reach critical size. This phase transition in the pre-critical nucleus allows us to quantify what value of the bcc-fluid interfacial free energy is needed in order to compensate for the difference in chemical potential of the two bulk structures. From our free-energy calculations, we deduce 
$\mu_{\mathrm{bcc}}-\mu_{\mathrm{fcc}}=0.082( \pm 0.01) k_{B} T$. We used the CNT expression for the barrier height to estimate the fcc-liquid interfacial free energy: $\gamma_{\mathrm{fcc}}=0.45 k_{B} T / \sigma^{2}$. The transformation from bcc to fcc nuclei occurred for $n \approx 100$. At that point, the gain in bulk free energy is $100 * 0.082=8.2 k_{B} T$. This free-energy gain must be compensated by the increase in surface free energy as the crystallite transforms from bcc to fcc. To estimate this surface free energy, we need to know the radius of the crystal nucleus for $n=100$. If we assume that the nucleus is spherical and that the solid is effectively incompressible, we arrive at the estimate $\gamma_{b c c}=0.38 k_{B} T / \sigma^{2}$. We find such a pre-critical transformation from bcc to fcc for $\beta \epsilon=2$ with $\kappa=5$, and for $\beta \epsilon=8$ with $\kappa=10$ and 3.33333. In all the other cases $(\beta \epsilon=6,8,20$ with $\kappa=5)$ even the critical nuclei had a strong bcc signature. This observation has implications for the interfacial free energies shown in Figs. 22 and 23. In these figures, we show interfacial free energies that were computed from the CNT expression for the barrier height, assuming that the nucleus had the same structure as the stable crystal phase. We now see that, in some cases, the critical nucleus has a meta-stable bcc structure. This affects the value for $\Delta \mu$ in the CNT expression, and hence our estimate for $\gamma$. In the cases where the critical nucleus has a bcc structure, we therefore also estimated the value of $\gamma_{\text {bcc }}$ from the height of the nucleation barrier. The results are also shown in Figs. 22 and 23.

Thus far we did not mention the possibility that the structure of the crystal nuclei could also be hexagonal closed packed (hcp) or a random stacking of the fcc and hcp domains (rhcp). In the case of hard-spheres it is known that the free-energy difference between the stable fcc and hcp solid structure is very small $\left(\approx 10^{-3} k_{B} T\right)$ and therefore stacking faults are expected. Such stacking faults have been observed in experiments and computer simulations. In the case of charged spheres the situation is less clear. Some experiments indicate that the situation changes and there seems to be tendency that crystal nuclei become more fcc-like [74]. Other experiments suggest that the structure of the cluster is still rhcp [60]. To resolve this question for the present model system, we first calculated the free energy difference between the fcc and hcp solid, for all the different parameters of the model potential for which we performed the rate calculations. It turns out that the free energy difference per particle between the fcc and hcp structure was always smaller than $1 \times 10^{-2} k_{B} T$ (see Table 4), which is about the limit of the accuracy that we had in our calculations. This means that thermal fluctuations on the order of a few $k_{B} T$ could easily transform clusters containing hundreds of particles from fcc to hcp, or generate intermediate stackings. To find out if this really happens we, analysed the stacking of the (111)planes of 10 nuclei with parameters $\beta \epsilon=8, \kappa=10$ and $\beta \epsilon=8, \kappa=3.33333$. In both cases, we do find stacking faults, but they seem to be less frequent than in the pure hard-sphere case. We stress, however, that these preliminary conclusions are based on the analysis of only a small number of crystallites. 


\section{6}

\section{Poly-12-hydroxystearic Coated PolymethyImethacrylate Particles}

Our findings for the weakly charged colloids suggest that even a slight "softness" of the intermolecular potential, has important consequences for the crystallization behavior. This effect could be relevant for experimental "hard-sphere" colloids, as these particles are, in fact, slightly soft. A particularly popular experimental hardsphere colloid consists of a polymethylmethacrylate (PMMA) core coated with a thin layer of poly-12-hydroxystearic (PHSA). Due to the coating, the particles are slightly soft. We studied the effect that such a softness has on the phase behavior and the crystallization kinetics [75].

The potential that we used to model the interaction between two PHSA-coated PMMA spheres was deduced from surface-force measurements. Costello et al. [76, 77] measured the force between two mica surfaces coated with a PMMA (backbone)PHSA (sidechain) comb copolymer, with the PMMA backbone directly adsorbed on the mica and the PHSA side chains protruding into the solvent. The interaction thus mimics that between the surfaces of two PHSA-stabilized PMMA colloids. Costello et al. analyzed their measurements according to a model proposed by Alexander and de Gennes [78]. In this model, expected to be valid for high grafting densities, each chain is assumed to consist of connected semi-dilute blobs. The chains are stretched by osmotic repulsion between the blobs. This tendency is opposed by the increase in elastic free energy of the chain upon stretching. The resulting expression for the force per unit area between two parallel plates at a distance $r$ is

$$
F(r)=\frac{\alpha k_{B} T}{s^{3}}\left[\left(\frac{2 L}{r}\right)^{9 / 4}-\left(\frac{r}{2 L}\right)^{3 / 4}\right],
$$

where $s$ is the mean spacing between between grafting points and $L$ is the thickness of the polymer layer; $\alpha$ is a numerical prefactor and $k_{B} T$ the thermal energy. The expression is supposed to hold for $0<r<2 L$. Integration yields the corresponding energy density. From the distance of onset of the interaction, Costello et al. estimated that their layer thickness was $L=12.5 \mathrm{~nm}$. A fit of the Alexander-de Gennes model to experimental measurements yielded $\alpha=0.025$ and $s=2.8 \mathrm{~nm}$. By using the Derjaguin approximation (see e.g. Ref. [79]) we can estimate the interaction potential between two spheres. Different methods have been used to measure the thickness of the PHSA layer on PMMA colloids synthesized according to the method of Antl et al. [80], giving values of $L=7-13 \mathrm{~nm}$ [81] and a maximum distance between grafting points of $s=2.0 \mathrm{~nm}$ [82]. As a starting point in our calculations, we used $L=13.5 \mathrm{~nm}$ and $s=2.0 \mathrm{~nm}$ to yield the strongest repulsion compatible with these experimental data. Denoting the radius of a particle's PMMA core (without the PHSA hair) as $R$, we plot the interaction potentials for two cases, $R=305 \mathrm{~nm}$ and $201 \mathrm{~nm}$, in Fig. 28. These two radii are chosen to enable us to compare our calculations with the equilibrium phase behavior data of Pusey and van Megen [29, 83] and the crystallization kinetics data of Harland and van Megen [5] respectively. 


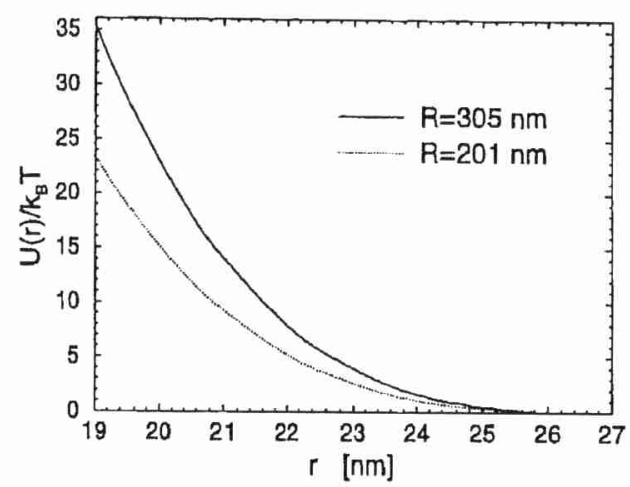

Fig. 28. Estimated interaction potential between two PMMA spheres coated with a layer of PHSA. Results are shown for particles with core radii of $R=201 \mathrm{~nm}$ and $305 \mathrm{~nm}$ with the following values for the parameters: $s=2.0 \mathrm{~nm}, L=13.5 \mathrm{~nm}, \alpha=0.025$

We see that in both cases the interparticle interaction increases steeply to $10 k_{B} T$ within 6-7 nm from the point of first contact.

We used the potential obtained in the previous section to calculate the freezing and melting densities of the colloidal suspensions from simulations using thermodynamic integration [15]. The resulting freezing and melting core volume fractions for our model potential were estimated to be $\phi_{f}=0.4137$ and $\phi_{m}=0.4579$ (for $R=201 \mathrm{~nm}$ ) and $\phi_{f}=0.4380$ and $\phi_{m}=0.4850$ (for $R=305 \mathrm{~nm}$ ). By scaling the freezing volume fractions to that of hard-spheres $\phi_{f}^{H S}=0.494$ [27] we obtain the effective hard-sphere diameter $\sigma_{\text {eff }}=1.061 \sigma$ and $\sigma_{\text {eff }}=1.041 \sigma$ of the two systems. We can compare these diameters to the effective hard-sphere diameter predicted by first order perturbation theory: $\sigma_{\text {eff }}=\int_{0}^{\infty} d r\left[1-\exp \left[-U(r) / k_{B} T\right]\right]$. The results $\sigma_{\text {eff }}=1.061 \sigma\left(\right.$ for $R=201 \mathrm{~nm}$ ) and $\sigma_{\text {eff }}=1.041 \sigma$ (for $R=305 \mathrm{~nm}$ ) are identical to the estimate above. The values for the interaction potential at this distance are $U\left(r=\sigma_{\text {eff }}\right) / k_{B} T=0.7056$ and 0.7065 . If we use the effective hard-sphere diameter to rescale the melting volume fractions of the soft systems to that of the hard spheres we find $\phi_{m}=0.5469$ and $\phi_{m}=0.5463$ (to be compared with $\phi_{m}^{\mathrm{HS}}=0.545$ [27]).

Our results can be compared directly with the observations of Pusey and van Megen $[29,83]$. These authors measured the core radius of their PMMA particles by static light scattering and electron microscopy, and found $R=305 \mathrm{~nm}$. Knowing the core radius $R$, Pusey and van Megen dried down their suspensions and converted the measured mass fraction to core volume fractions using literature values of the densities of PMMA and the suspending liquid. They found core volume fractions at freezing and melting $\phi_{\mathrm{f}}=0.407$ and $\phi_{\mathrm{m}}=0.441$ [47]. The corresponding effective hard sphere diameter is $\sigma_{\text {eff }}=1.067$. The experimental volume fractions are some $3.1 \%$ lower than the freezing volume fraction determined in our simulations. If we consider the fact that the particles are polydisperse (5\%) the discrepancy increases to $4.1 \%$ [52]. One may seek to obtain a better fit to experiments by varying the parameters $s$ and $L$. The value of $s$ used gives the minimum surface coverage (at 
areal density $s^{-2}$ ) necessary for steric stabilization to function [82]. In any case, we find that the effective hard sphere diameter is somewhat insensitive to variations in $s$. Instead, agreement with the hard-core freezing volume fraction of Pusey and van Megen can be obtained by using a value of $L \approx 22 \mathrm{~nm}$. While there was no

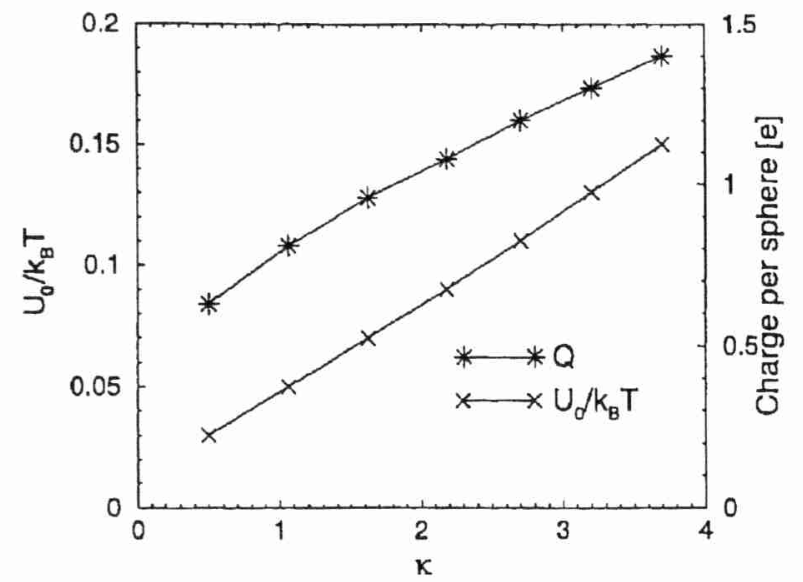

Fig. 29. Calculated parameter set $\left(U_{0} / k_{B} T, \kappa\right)$ of a hard-core Yukawa potential that accounts for the observed shift in the freezing density. The same curve but in units of charge per sphere is also shown

direct determination of the PHSA chain length for the batch of PMMA particles used by these authors, this value of $L$ is twice to three times as long as values obtained from a variety of experiments on PHSA-coated PMMA particles [81]. Pusey and van Megen, who estimated the effective hard sphere diameter of their particles to be $\sigma_{\text {eff }}=2 R \times(0.494 / 0.407)^{1 / 3}$, also concluded [83] that the implied PHSA layer thickness of $L \sim 20 \mathrm{~nm}$ was rather larger than expected. It is therefore possible that there is an additional source of weak repulsion, such as a slight charge on the colloids. If we assume that the interaction between charged colloids is described by a repulsive hard core Yukawa potential: $U_{0} / k_{B} T \exp [-\kappa(r / \sigma-1)] /(r / \sigma)$ for $r>\sigma$, we can use the previous equation for the effective hard-sphere diameter from first order perturbation theory to estimate the values of the parameter $U_{0} / k_{B} T$ and $\kappa$ needed to account for the observed shift in freezing volume fraction. Here $U_{0} / k_{B} T$ is the value of the Yukawa repulsion at contact and $\kappa$ is the inverse screening lenght in units of the hard-sphere diameter $\sigma$. We find that the added repulsion is indeed quite weak, and very soft (see Fig. 29). Note that such a weak, soft repulsion can hardly be detected in the surface-force measurement. We can estimate the charge on a particle from the contact value of the interaction potential: $U_{0} / k_{B} T=Q^{2} / 4 \pi \epsilon_{0} \epsilon \sigma$, where $Q$ is the charge, $\epsilon_{0}$ and $\epsilon$ are the permittivity of the vacuum and the solvent. A value $U_{0} / k_{B} T=0.1$ corresponds to an average colloidal charge of about one electron per sphere. More recent experiments by Bryant et al. [84] indicate that the polymer layer 
thickness is even smaller, which suggests that the charge might even be higher. In more polar solvents, long range repulsions have been observed for the same kind of particles [85].

We turn now to study how the softness of the potential affects the crystallization kinetics. For the system with $R=201 \mathrm{~nm}$ we computed the crystal nucleation barrier at four different pressures $P \sigma^{3} / k_{B} T=12.5,13,13.5$ and 14 , corresponding to volume fractions of the liquid $\phi_{l}=0.43441,0.43803,0.44144$ and 0.44480 . In Fig. 30 we show the results for the crystal nucleation barrier as a function of $\Delta \mu$. In the figure we also show the results for the hard-sphere system. As can be seen, despite the only slight softness, the crystal nucleation barrier is reduced by about 2$4 k_{B} T$ at constant $\Delta \mu$. This is largely the result of a lowering of the surface tension compared to the case of hard spheres. If we assume that the nuclei are spherical we can use Eq. (2) to calculate the surface free energy density of the critical nuclei. The results are $y=0.592,0.608,0.629,0.636 k_{B} T / \sigma^{2}$ (in order of increasing density).

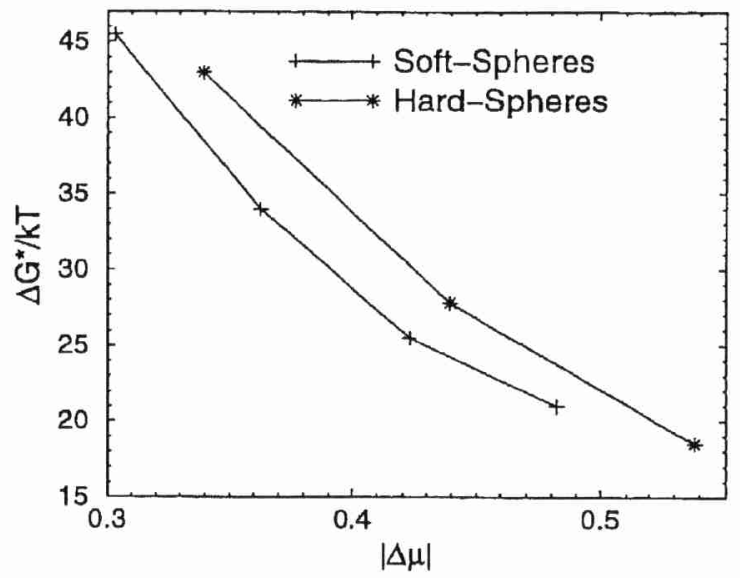

Fig. 30. Computed crystal nucleation barrier heights $\Delta G^{*}$ for the slightly soft-spheres plotted as a function of supersaturation $\Delta \mu$. In addition we also show results from previous simulation on the hard-sphere system

To estimate the crystal nucleation rate we computed the kinetic prefactor $\Gamma$ as described before. The result for the crystal nucleation rates as a function of $\Delta \mu$ is that the decrease in the nucleation barrier transforms into an increase of the crystal nucleation rate of about two orders of magnitudes. Our simulations can be compared directly with the experimental results of Harland and van Megen [5], who measured nucleation rates by time-resolved static light scattering for PMMA spheres of radius $201 \mathrm{~nm}^{1}$. To make this comparison, we show in Fig. 31 the crystal nucleation rate as a function of the rescaled volume fraction of the metastable fluid. Comparing

${ }^{1}$ Essentially, this radius was determined by assuming the hard-sphere freezing and melting volume fractions of 0.494 and 0.545 respectively. Thus $201 \mathrm{~nm}$ is not the core radius (but 
first the results for monodisperse hard spheres [32] and monodisperse soft spheres (this work), we see that there is again an increase of the nucleation rate of more than one order of magnitude. However, the particles used in Harland and van Megen were $5 \%$ polydisperse. Previous simulation results for $5 \%$ polydisperse hard spheres [32] are reproduced in Fig. 31: these disagree with Harland and van Megen's data by up to 10 orders of magnitude. If we assume that the effect of softness on the nucleation rate is also an upward shift of a little over an order of magnitude, then results for polydisperse soft spheres would agree somewhat better with the data, but substantial disagreement remains. We also show the results of experiments by Sinn et al. [31]. The particles they used are larger $(435 \mathrm{~nm}$, and therefore less soft) and have a polydispersity of $2.5 \%$ (i.e. more monodisperse than the particles used by Harland and van Megen). The simulation results for monodisperse hard spheres can therefore be expected to be more comparable. Even here, however, there remains many orders of magnitude disagreement.

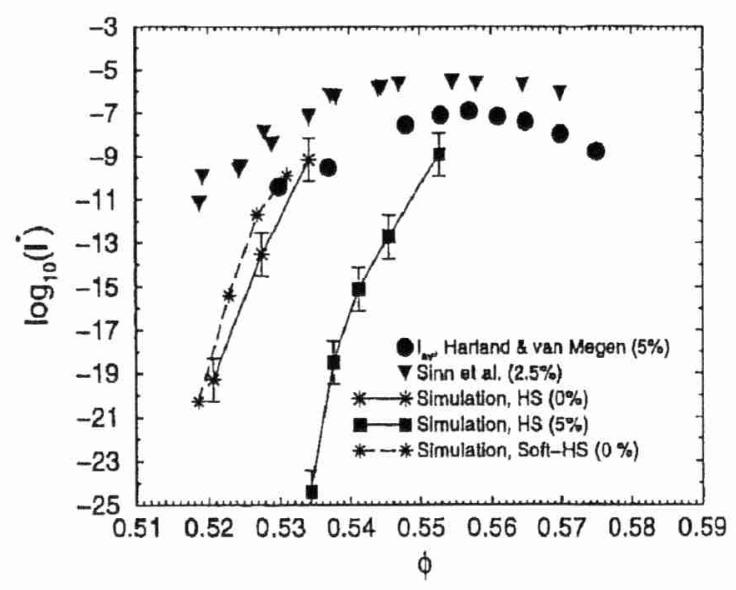

Fig. 31. Reduced nucleation rates $I^{*}$ as a function of the rescaled volume fraction of the meta-stable fluid. We show the experimental results of Ref. [5] ( $\bullet$ and Ref. [31] ( $)$. The simulation data for the monodisperse colloids are indicated by $(*)$, where the solid and the dotted lines correspond to the hard sphere system and the slightly soft system. The results of the hard sphere system that has a polydispersity of $5 \%$ are shown as (I)

The fact that the particles may be weakly charged and the system has a large Debye screening length might have two additional effects on the crystallization kinetics. First of all, the charge further lowers the surface free energy which increases the nucleation rates. Secondly, as both the surface charge and the Debye screening

more like $R+L$ ). We nevertheless have estimated the interparticle potential in this case using $R=201 \mathrm{~nm}$, because the accuracy of our nucleation barrier simulations and the uncertainties associated with estimating the absolute nucleation rates do not warrant any attempt at estimating $R$ to an accuracy of $5 \%$. 
length may depend on density this can qualitatively change the dependence of the nucleation rate on supersaturation [61]. A better agreement with experimental nucleation rates would be obtained if we make the (not unreasonable) assumption that the colloids become more hard-sphere like at higher densities.

\section{7}

\section{Wall Induced Crystallization}

Thus far, we have focused on the homogeneous nucleation in colloidal suspensions. However, in the real world, crystallization is usually initiated by heterogeneous nucleation. If ice could only form through homogeneous nucleation, the freezing of water would be a rare phenomenon in countries with moderate climates.

To study the effect of an external surface on the crystallization process, we studied the behavior of monodisperse hard-sphere colloids near a hard wall [86]. Depending on the nature of the surface, it may have different effects on the freezing transition. One possibility is that the crystal phase "wets" the surface: in that case, one or more crystalline layers form at the surface, before the bulk freezing transition. Alternatively, the crystal may partially wet the wall, in which case crystal nucleation from a supersaturated solution takes place at the wall, rather than in the bulk.

The effect of a structured surface on the crystallization of hard-sphere colloids has been extensively studied in experiments $[87,88,89,90]$. These experiments indicate that crystallization on a template is induced at densities below freezing. This finding is supported by computer simulations of hard spheres in contact with a patterned substrate, by Heni and Löwen [91]. These simulations indicate that surface freezing already sets in $29 \%$ below the coexistence pressure. Furthermore the effect of a surface on crystallization has also been studied in mixtures of binary hardspheres $[92,93]$ and colloid-polymer mixtures $[94,95,96]$. In both systems surface crystallization was found to take place before bulk fluid-solid coexistence. In the systems studied in Refs. [92, 93, 94, 95, 96], depletion forces favor the accumulation of the larger component on the wall, and this should facilitate surface crystallization [97].

For the important case of pure hard-sphere systems confined by flat walls, it is not a priori clear if bulk freezing will be preceded by surface crystallization. Yet, we are not aware of any systematic, experimental studies of surface crystallization in pure hard-sphere systems. Courtemanche and Swol [98] reported a numerical study of a (rather small) one-component hard sphere system, confined between two plane hard walls. These simulations suggested that surface crystallization occurred at a pressure some $3 \%$ below the coexistence value.

Before we present the simulation results, we briefly discuss the effect of a wall on crystal nucleation in the context of Classical Nucleation Theory (CNT). Turnbull [99] extended CNT to the case of heterogeneous nucleation of a crystal that forms on a plane substrate. The difference with the homogeneous case is that there are now two interfaces present. The Gibbs free energy of a crystal containing $n$ particles is given by: 


$$
\Delta G(n)=n \Delta \mu+A_{w s}\left(\gamma_{w s}-\gamma_{w l}\right)+A_{l s} \gamma_{l s},
$$

where the subscripts $w, l, s$ refer, respectively, to the wall, the liquid and the solid. Note that in this formulation the contribution to $\Delta G(n)$ due to the line tension is neglected. More seriously, the dependence of the interfacial free energy on the surface orientation is ignored. With those assumptions, the shape that minimizes $\Delta G(n)$ at fixed $n$, is a sphere sector, with a contact angle $\theta$ of the two phases with the wall given by: $\cos (\theta)=\left(\gamma_{w l}-\gamma_{w s}\right) / \gamma_{l s}$. The resulting height of the nucleation barrier is

$$
\Delta G^{*}=\frac{16 \pi}{3} \frac{\gamma_{l s}^{3} f(\theta)}{\left(\rho_{s} \Delta \mu\right)^{2}},
$$

where $\rho_{s}$ is the number density of the bulk solid and the factor $f(\theta)=(2+$ $\cos (\theta))(1-\cos (\theta))^{2} / 4$. The only difference with the expression for the homogeneous case is the factor $f(\theta)$. Depending on the values for the interfacial free energy densities, we distinguish three different cases. The first case corresponds to the situation where $\gamma_{w s}>\gamma_{w l}+\gamma_{s l}$. Under these conditions the crystal will not form on the substrate, because this would increase its free energy, and nucleation will take place in the bulk. A second scenario applies when $-\gamma_{l s}<\gamma_{w l}-\gamma_{w s}<\gamma_{l s}$. Then $-1<\cos (\theta)<1$. This means that a crystal can lower its free energy by attaching to the wall (partial wetting). The final case is when $\cos (\theta)=1\left(\theta=0^{\circ}\right)$ or $\gamma_{w l}>\gamma_{w s}+\gamma_{l s}$. In that case, the solid phase prefers to form a thin layer on the wall (complete wetting) and the barrier to nucleation disappears.

For the hard-sphere system, we can speculate what scenario should apply, as all relevant surface free energies have been estimated numerically $[100,7]$, at least at coexistence. The estimated value for the wall/liquid interfacial free energy density at the freezing volume fraction $\phi=0.494$ is $\gamma_{w l}=1.99 k_{B} T / \sigma^{2}$ [100], where $\sigma$ is the hard-sphere diameter and $k_{B} T$ the thermal energy. The values for the wall/solid interfacial free energies for different orientations (111), (110), (100) are estimated to be $\gamma_{w s}=1.42,3.08,2.01 k_{B} T / \sigma^{2}$ [100]. The values for the liquid/solid interfacial free energy for the same three orientations are $\gamma_{l s}=0.58,0.64,0.62 k_{B} T / \sigma^{2}$ [7]. These numbers suggest that the (110) plane will not attach to the wall as $\gamma_{w l}+\gamma_{l s}<$ $\gamma_{w s}$. In contrast, the (100) planes is expect to partially wet the wall. For the (111)plane, the difference between $\gamma_{l s}+\gamma_{w s}$ and $\gamma_{w l}$ is estimated to be $0.01 \pm 0.18$, which is not significantly different from zero. Hence, the (111) plane is expected to be at, or very close to, complete wetting.

To explore the pathway for wall-induced crystallization, we performed Monte Carlo simulations in the constant normal-pressure $\left(N P_{\perp} T\right)$ ensemble. Here $N$ refers to the number of hard-spheres in the system. The simulation box was rectangular with periodic boundary conditions in the $x$ and $y$ directions. In the $z$-direction, the system is confined by two flat, hard walls at a distance $L_{z} . P_{\perp}$ is the component of the pressure tensor perpendicular to the plane wall, and $T$ is the temperature. As our unit of length we used the hard-sphere diameter $\sigma . T$ only sets the energy scale. In the following we always use reduced units. The state of the bulk hard-sphere system is completely specified by its volume fraction $\phi$. The coexistence volume fractions for the bulk fluid and solid phase are known [27]: $\phi_{f}=0.494$ and $\phi_{m}=0.545$. The 
corresponding coexistence pressure is $P_{c}=11.57$. To suppress finite-size effects, we simulated a system containing $N=13824$ particles. The wall area was fixed at $L_{x} L_{y}=600.25 \sigma^{2}$, the distance between the two walls in the $z$-direction fluctuated but was close to $24 \sigma$, which is much larger than any correlation length in the fluid. During the simulations, we performed on average one volume move per two cycles (trial moves per particle).

The simplest way to detect if a crystal phase wets the surface is to measure the density profile of the particles between the two walls. In Fig. $32 \mathrm{a}$ we show the ob-

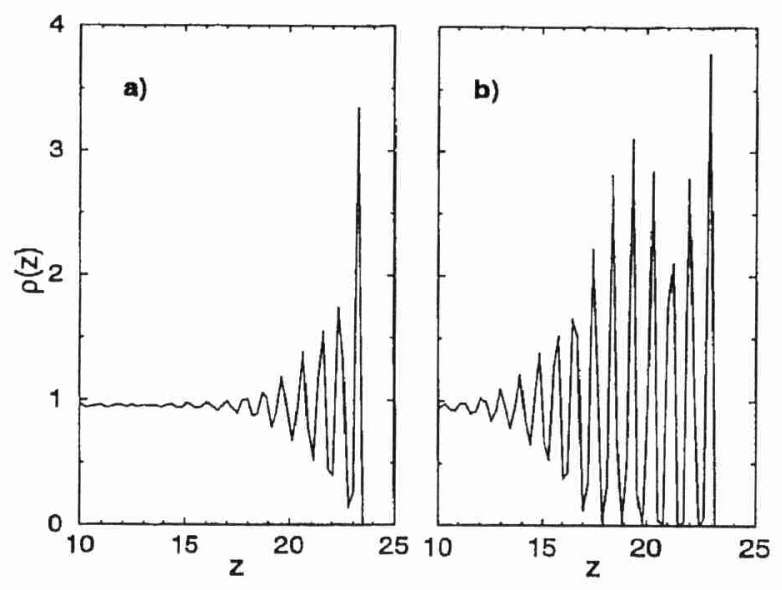

Fig. 32. (a) Density profile along the $z$-direction for a hard-sphere system between the two plane walls at an excess pressure $\Delta P=0.53$. The corresponding bulk volume fraction is $\phi=$ 0.4966 . Simulation length: $2 \cdot 10^{6}$ cycles. (b) as in (a) but at an excess pressure $\Delta P=0.63$

served density profile at the end of a simulation performed at a pressure just above bulk freezing (excess pressure $\Delta P \equiv P_{\perp}-P_{c}=0.53$ ). If crystallization at the wall had taken place, this would cause a pronounced dip between the first and the second peak in Fig. 32a. No such behavior was observed, even at pressures well above $P_{c}$.

The situation changes when the excess pressure is increased to $\Delta P=0.63$. The liquid starts to crystallize, as can be seen from the density profile shown in Fig. 32b. These results indicate that supersaturation is needed to induce crystallization. Yet, the degree of supersaturation needed to induce nucleation is very small compared to that typical for bulk systems. In fact, in simulations of homogeneous systems of comparable size, the rate of crystal nucleation during a simulation of similar length, is negligible up to excess pressures that are an order of magnitude larger $(\triangle P \approx 5.4$ $(\phi \approx 0.53)$ ). In order to identify the early stages of crystal nucleation, we used a local bond-order analysis [32] to distinguish between particles with a liquid-like and solid-like local environment. The result of this analysis is shown in Fig. 33, which shows a snapshot of the particles closest to the wall at $\Delta P=0.53$. The dark particles have a liquid-like environment and the light particles have a solid-like environment. 


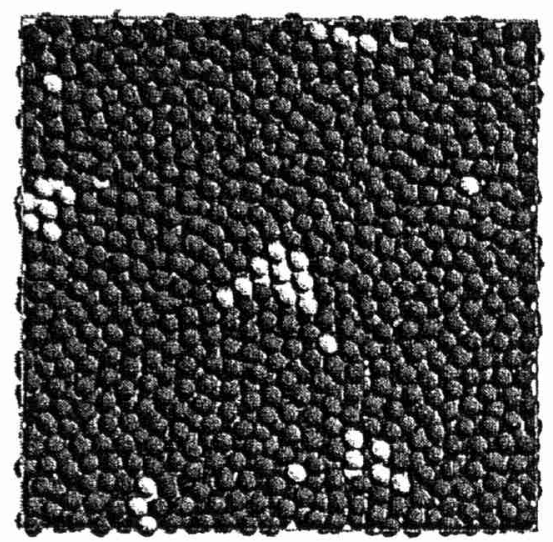

Fig. 33. Snapshot of a configuration which shows the particles at the wall. A local bondorder analysis was used to distinguish between particles with a liquid-like (dark particles) and solid-like (light particles) environment. The snapshot is taken from a simulation at pressure $P_{\perp}=12.1$

Only a few small crystalline clusters can be identified. These clusters form and break up spontaneously. Under the same conditions, not a single solid-like cluster formed in the bulk of the fluid.

A more quantitative measure of the effect of the surface on crystal nucleation we obtained from a direct calculation of the crystal-nucleation barrier. We performed Monte Carlo simulations in the constant normal-pressure $\left(N P_{\perp} T\right)$ ensemble where we used $N=13824$ particles and simulated $2 \cdot 10^{6}$ cycles for every window. The result for the free energy barrier calculated at a pressure $\Delta P=0.63$ is shown in Fig. 34 (dots). At this pressure, the estimated barrier height is $\Delta G^{*}=17 k_{B} T$ at a critical cluster size $n_{c}=150$.

We can compare this estimate with a prediction for the barrier height in a homogeneous system. For the hard-sphere colloids we showed before that, given the correct value for the interfacial free energy, CNT describes the barrier height quite well [32]. But we also found that the interfacial free energy depends on density. As the present system is close to coexistence we use its average coexistence value $\gamma_{a v}=0.61 k_{B} T / \sigma^{2}$ [7]. We then obtain $\Delta G_{C N T}^{*}=1334 k_{B} T$ at a critical cluster size of $n_{c}=52000$. The overall reduction of the nucleation barrier due to the plane wall is about two orders of magnitudes, resulting in a huge $\left(O\left(10^{570}\right)\right)$ increase in the nucleation rate. The computed nucleation rate per unit area is $\sim 10^{-9}$ (in units $\left.D_{0} / \sigma^{4}\right)$.

The implication for experiments is clearly that crystallization of suspensions of hard-sphere colloids should proceed heterogeneously, whenever a sufficiently flat surface is available. Yet, somewhat surprisingly, there are, to our knowledge, no systematic experimental observations of surface-induced freezing in hard sphere colloids, even though most bulk crystallization studies are performed in contain- 
ers with, effectively flat walls. When we compare the computed nucleation barrier

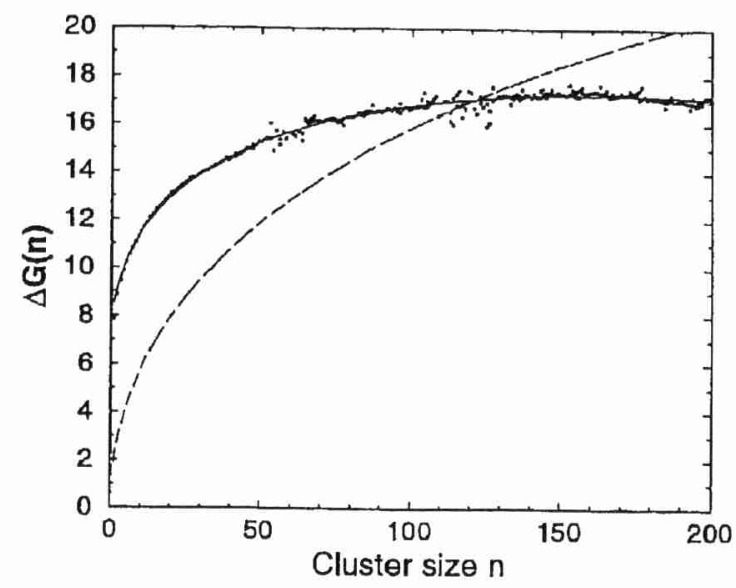

Fig. 34. Calculated nucleation barrier $\Delta G(n)$ of a crystal nucleus formed at the wall as a function of its size $n$ (filled dots). In the figure, we show two fits to the nucleation barrier: the dashed curve assumes the published values for the surface free energies and uses a curvatureindependent line tension. To obtain the drawn curve, we used $\gamma_{w l}$ as a fit parameter and we assumed that the line-tension was curvature dependent. If we had used the CNT expression (Eq. 15), there would not be a nucleation barrier at this supersaturation

with the predictions of CNT (Eq. (15)), we find that this expression seriously underestimates the height of the nucleation barrier. In fact, CNT would predict that, at an excess pressure $\Delta P=0.53$ ), (where $\Delta \mu=-0.05 k_{B} T$ [33]), the barrier to nucleation is negligible compared to $k_{B} T$. In order to resolve this discrepancy, we are forced to take into account the line tension, $\tau_{\text {Line }}$, of the crystal nuclei on the wall. If we attempt to fit our numerical data to Eq. (15) plus a term due to line tension, we can indeed reproduce a nucleation barrier with the same height as found in the simulations, but the shape of the simulated barrier is reproduced rather poorly (see Fig. 34). A much better fit can be obtained by allowing $\gamma_{w l}$ to vary within the bounds set by the (large) estimated error in the computed value: $1.99( \pm 0.18) k_{B} T / \sigma^{2}$. In addition, it turns out that we have to allow for a curvature correction of the line tension: $\tau_{\text {Line }}=\tau_{\infty}+c / R$. This fit yields $\tau_{\infty}=0.43 k_{B} T / \sigma, c=1.1 k_{B} T$ and $\gamma_{w l}=2.016 k_{B} T / \sigma^{2}$. Note that with this value of $\gamma_{w l}$, the condition for complete wetting would be satisfied $\gamma_{w s}+\gamma_{s l}-\gamma_{w l}=-0.02 k_{B} T / \sigma^{2}$. This would agree with the conclusion of Ref. [98]. However, the statistical inaccuracy in this estimate is appreciable. We can compare our fitted value for $\tau_{\infty}$ with a naive estimate by assuming that the contribution to the free energy due to line tension is really nothing else than the surface free energy of the lateral surface of a cylinder of height $1 \sigma$. Assuming that the lateral surface free-energy density is approximately equal to $\gamma_{l s}^{(110)}$, our estimate for $\tau_{\infty}$ would be $\tau_{\infty} \approx 0.64 k_{B} T / \sigma$, which is within $50 \%$ of the numerical 
result. An estimate of the curvature correction to $\tau_{\text {Line }}$ would necessarily be even cruder. From the simulations, we can also determine the orientation and shape of the

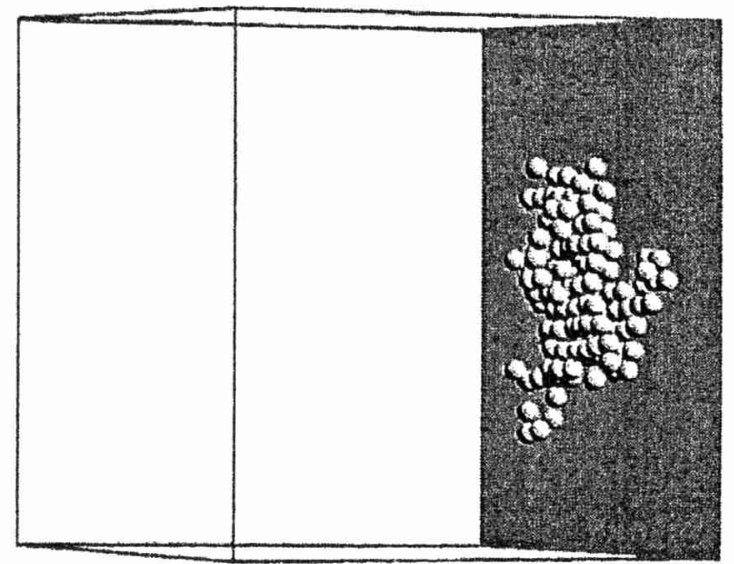

Fig. 35. Sideview of the snapshot of a crystal nucleus of size $n=150$

incipient crystal nucleus. Figure 35 shows a snapshot of a critical nucleus containing 150 particles. From the figure, it is clear that the (111) plane attaches to the wall. Note that the critical nucleus is quite flat. Clearly, small nuclei prefer to spread on the surface rather to grow into the bulk. This is in agreement with the CNT predictions in the case where the (111) face wets the wall, either completely or very nearly so.

The fact that the range of metastability becomes very narrow might provide a powerful tool for the determination of the freezing density in experiments. Using confocal microscopy it should be possible to detect the formation of crystallites on a flat surface. Provided the interaction of the particles with the wall is the same as the interparticle potential, such crystallites will be first observed under conditions where the bulk density differs less than $1 \%$ from its value at coexistence. Our simulations suggest that pre-freezing first occurs at a pressure that is some $2 \%$ below the coexistence pressure, but, as explained above, this estimate is subject to a large statistical uncertainty.

\section{8}

\section{Concluding Remarks}

Computer simulations of crystal nucleation play a dual role. On the one hand, they can be used as a direct test of existing nucleation theories and, on the other, they can be compared directly with experiments (provided we have a good model for the experimental system). The fact that both types of comparisons lead to discrepancies, is 
interesting. It suggests that the existing nucleation theories may need to be improved, and it indicates that there is something wrong with our interpretation of nucleation experiments. There may be a problem with our model, or with our assumption about the experimental conditions ("steady-state, homogeneous nucleation"). Of course, there may also be problems with our numerical approach. It is appropriate to consider the latter possibility in this paragraph. As we discussed in the text, there is a certain degree of arbitrariness in the choice of the order parameter that measures crystallinity. Hence, the reported size of the crystal nuclei should be taken with a grain of salt. However, as long as the real size of the nucleus is related linearly to the computed size, the height of the nucleation barrier is not affected by a different choice of order parameter. Any estimate of the surface free energy that is based on this height, is therefore also insensitive to the choice of order parameter. However, if we use the complete shape of the nucleation barrier to compute the surface free energy, then we may expect to find that the results depend on the choice of order parameter. In fact, this is not surprising, as the surface free energy of a spherical object necessarily depends on our choice for the location of the surface (e.g. surface of tension or equimolar surface). Finally, the nucleation rate should not depend at all on our choice of order parameter: this is a true, physical observable that cannot depend on the scheme that we use to compute it.

We should always bear in mind that the Classical Theory of Nucleation is, in essence, a macroscopic theory. But, at the microscopic level, such a level of description is not adequate. In the end, all observable quantities should be expressed as functions of material properties that are, themselves, unambiguously observable.

\section{Acknowledgements}

We gratefully acknowledge Alfons van Blaaderen and Thomas Palberg for helpful discussions. Thomas Palberg we thank for permission to show Fig. 3 and Evert Jan Meijer for permission to show Fig. 19. The work of the FOM Institute is part of the research program of FOM and is made possible by financial support from the Netherlands Organization for Scientific Research (NWO).

\section{A}

\section{Distribution of Cluster Sizes in Equilibrium}

The distribution of cluster sizes can be derived microscopically from statistical mechanics. The derivation is based on Refs. $[10,101,9]$. The partition function of a system containing $N$ particles in a volume $V$ at temperature $T$ is given by

$$
Q(N, V, T)=\frac{1}{\Lambda^{3 N} N !} \int d \mathrm{r}^{N} \exp \left[-\beta U\left(\mathrm{r}^{N}\right)\right] .
$$

Here $U\left(\mathrm{r}^{N}\right)$ is the potential energy of the configuration with coordinates $\mathrm{r}^{N}$ and $\Lambda=h / \sqrt{2 \pi m k T}$ is the thermal de Broglie wavelength. Now we assume that we 
have a criterion, that enables us to identify a cluster in our system. We then define a function $w_{n}\left(\mathrm{r}^{n}\right)$ such that

$$
w_{n}\left(\mathrm{r}^{n}\right)= \begin{cases}1 & \text { if all } n \text { particles belong to the cluster } \\ 0 & \text { otherwise }\end{cases}
$$

In addition, we define a function $w_{r}\left(\mathbf{r}^{N}\right)=\prod_{i=n+1}^{N}\left[1-w_{n+1}\left(\mathbf{r}^{n}, \mathbf{r}_{i}\right)\right]$, which ensures that all other particles do not belong to the cluster

$$
w_{r}\left(\mathrm{r}^{N}\right)= \begin{cases}1 & \text { if no other particle belongs to the cluster } \\ 0 & \text { if any other particle belongs to the cluster }\end{cases}
$$

We can then define a partition function for a system that contains at least one $n$ particle cluster

$$
\begin{aligned}
& Q_{n}(N, V, T)=\frac{1}{A^{3 n} n !} \frac{1}{A^{3(N-n)}(N-n) !} \times \\
& \quad \int d \mathbf{r}^{n} \int d \mathbf{r}^{N-n} w_{n}\left(\mathbf{r}^{n}\right) w_{r}\left(\mathbf{r}^{N}\right) \exp \left[-\beta U\left(\mathbf{r}^{n}, \mathbf{r}^{N-n}\right)\right],
\end{aligned}
$$

where we have used the fact that there are $N ! /(n !(N-n) !)$ ways to select an $n$ particle cluster. Note that the remaining particles may still form additional clusters of size $n$. The product $w_{n}\left(\mathbf{r}^{n}\right) w_{r}\left(\mathbf{r}^{N-n}\right)=1$, only if all $\mathbf{r}^{n}$ particles belong to the specified cluster and all the other $\mathbf{r}^{N-n}$ do not. We now rewrite the potential energy of the system as the sum of contributions from the particles in the cluster $U_{n}\left(\mathbf{r}^{n}\right)$ and the contribution from all other particles $U_{N-n}\left(\mathrm{r}^{N-n}\right)$, plus the contribution from the interactions between particles in the cluster and the others $U_{n, N-n}\left(\mathbf{r}^{n}, \mathbf{r}^{N-n}\right)$. The partition function then becomes

$$
\begin{aligned}
& Q_{n}(N, V, T)= \\
& \quad \frac{1}{\Lambda^{3 n} n !} \frac{1}{\Lambda^{3(N-n)}(N-n) !} \int d \mathrm{r}^{N-n} \exp \left[-\beta U_{N-n}\left(\mathrm{r}^{N-n}\right)\right] \\
& \times \int d \mathrm{r}^{n} w_{n} w_{r} \exp \left[-\beta U_{n}\left(\mathrm{r}^{n}\right)\right] \exp \left[-\beta U_{n, N-n}\left(\mathbf{r}^{n}, \mathrm{r}^{N-n}\right)\right]
\end{aligned}
$$

We can now define effective potentials for all the particles in the cluster

$$
U_{n}^{\prime}=U_{n}-k T \ln \left[w_{n}\right],
$$

and the interaction between cluster particles and the others

$$
U_{n, N-n}^{\prime}=U_{n, N-n}-k T \ln \left[w_{r}\right],
$$

yielding

$$
\begin{aligned}
& Q_{n}(N, V, T)= \\
& \frac{1}{\Lambda^{3(N-n)}(N-n) !} \frac{1}{\Lambda^{3 n} n !} \int d \mathrm{r}^{N-n} \exp \left[-\beta U_{N-n}\left(\mathrm{r}^{N-n}\right)\right] \\
& \int d \mathrm{r}^{n} \exp \left[-\beta U_{n}^{\prime}\right] \exp \left[-\beta U_{n, N-n}^{\prime}\right] .
\end{aligned}
$$


Multiplication of the right side by $Q(N-n, V, T) / Q(N-n, V, T)$ gives

$$
\begin{aligned}
Q_{n}(N, V, T)= & \frac{1}{n ! \Lambda^{3 n}} Q(N-n, V, T) \\
& \int d \mathbf{r}^{n}\left\langle\exp \left[-\beta U_{n, N-n}^{\prime}\right]\right\rangle \exp \left[-\beta U_{n}^{\prime}\right],
\end{aligned}
$$

where we have defined a potential of mean force

$$
\begin{aligned}
& \left\langle\exp \left[-\beta U_{n, N-n}^{\prime}\right]\right\rangle= \\
& \frac{\int d \mathbf{r}^{N-n} \exp \left[-\beta U_{n, N-n}^{\prime}\right] \exp \left[-\beta U_{N-n}\left(\mathbf{r}^{N-n}\right)\right]}{(N-n) ! A^{3(N-n)} Q(N-n, V, T)} .
\end{aligned}
$$

It is the average potential the particles in the cluster feel due to the interactions with all other particles. We define now the partition function of an n-mer as

$$
q_{n}(V, T)=\frac{1}{n ! \Lambda^{3 n}} \int d \mathbf{r}^{n}\left(\exp \left[-\beta U_{n, N-n}^{\prime}\right]\right) \exp \left[-\beta U_{n}^{\prime}\right]
$$

Note that $q_{n}(V, T, \mu)$ is the partition function of a cluster in which the interaction with the remaining $(N-n)$ molecules is included in the factor $\left\langle\exp \left[-\beta U_{n, N-n}^{\prime}\right]\right\rangle$. The interaction with possible other clusters is also included as such clusters can still exist in the remaining $(N-n)$ particles. The partition function Eq. (18) can then be written as

$$
Q_{n}(N, V, T)=Q(N-n, V, T) q_{n}(V, T) .
$$

The probability to find at least one cluster of size $n$ is then given by

$$
P_{n}=\frac{Q_{n}(N, V, T)}{Q(N, V, T)}=\frac{Q(N-n, V, T)}{Q(N, V, T)} q_{n}(V, T) .
$$

As the free energy of the system is given by $F=-k T \ln [Q]$, the above equation becomes

$$
P_{n}=q_{n}(V, T) \exp [-\beta(F(N-n, V, T)-F(N, V, T))] .
$$

Using

$$
F(N-n, V, T) \approx F(N, V, T)-\left(\frac{\partial F}{\partial N}\right)_{V, T} n
$$

it follows that

$$
P_{n}=q_{n}(V, T) \exp [+\beta \mu n] .
$$

The problem with this definition of the probability is that it depends on the volume $V$. To see this we rewrite Eq. (18)

$$
q_{n}(V, T)=\frac{1}{n ! \Lambda^{3 n}} \int d \mathbf{r}^{n} \exp \left[-\beta U_{e f f}\right]
$$


where we defined an effective potential $U_{e f f}=\left\langle U_{n, N-n}^{\prime}\right\rangle+U_{n}^{\prime}$. Rewriting the partition function in terms of the center of mass of the cluster yields

$$
q_{n}(V, T)=\frac{n^{3}}{n ! \Lambda^{3 n}} \int d R_{C M} \int d \mathbf{r}^{\prime n-1} \exp \left[-\beta U_{e f f}\right] .
$$

Performing the integral over the center of mass and defining a partition function of the cluster in terms of the internal coordinates we get

$$
q_{n}=\frac{V}{\Lambda_{n}^{3}} \times q_{n}^{\text {internal }},
$$

where $A_{n}=h / \sqrt{2 \pi n m k T}$ is the de Broglie wavelength of the cluster and

$$
q_{n}^{\text {internal }}=\frac{n^{3 / 2}}{\Lambda^{3(n-1)} n !} \int d \mathbf{r}^{n-1} \exp \left[-\beta U_{\text {eff }}\right] .
$$

It is better to define an intensive probability distribution

$$
\frac{P_{n}}{N}=\frac{1}{\rho \Lambda_{n}^{3}} q_{n}^{\text {internal }} \exp [-\beta \mu n],
$$

where $\rho$ is the number density of the system. For rare clusters we can write the probability as

$$
P_{n}=p_{n}(1)+p_{n}(2)+\cdots \approx p_{n}(1),
$$

where $p_{n}(i)$ is the probability that there are exactly $i$ clusters of size $n$. If we assume that the formation of different clusters is uncorrelated $p_{n}(i)=\left[p_{n}(1)\right]^{i}$, then we can neglect higher order terms provided the probabilities are small, $p_{n}(1) \ll 1$. As the average number of clusters of size $n$ is equal to

$$
N_{n}=1 p_{n}(1)+2 p_{n}(2)+3 p_{n}(3)+\cdots
$$

we can write in the case of rare clusters

$$
\frac{P_{n}}{N} \approx \frac{N_{n}}{N}=\frac{1}{\rho \Lambda_{n}^{3}} q_{n}^{\text {internal }} \exp [-\beta \mu n]
$$

We note that this is a classical result and should not depend on Planck's constant $h$, and, in fact it does not, as the ideal gas part of the chemical potential

$$
\mu=\mu^{e x}+k T \ln [\Lambda]
$$

cancels the $h$ in $\Lambda_{n}$.

The main point of Eq. (21) is that we can write down a microscopic expression for the equilibrium number of $n$-clusters if this number, which is equal to the probability of finding one cluster of size $n$, is much less than one. Using Eq. (6) this in turn defines an intensive Gibbs free-energy of the cluster where the reference state is the homogeneous phase:

$$
\frac{N_{n}}{N}=\exp \left[-\Delta G(n) / k_{B} T\right] .
$$

This is the key relation which enables us to compute a nucleation barrier in a Monte Carlo simulation. 
B

\section{Calculation of the Chemical Potential}

Here we describe the calculation of the chemical potential for the monodisperse hardsphere system. For the calculation of the chemical potential of the two phases, we performed a thermodynamic integration. The Helmholtz free energy $F$, per particle and in units of the thermal energy $k_{B} T$, of a liquid is determined by integrating the equation of state, starting from low densities, where the fluid behaves like an ideal gas [1.5]:

$$
\frac{F(\rho)}{N k_{B} T}=\frac{F^{i d}(\rho)}{N k_{B} T}+\frac{1}{k_{B} T} \int_{0}^{\rho} d \rho^{\prime}\left(\frac{P\left(\rho^{\prime}\right)-\rho^{\prime} k_{B} T}{\rho^{\prime 2}}\right),
$$

where $P(\rho)$ is the pressure and $F^{i d}(\rho) / N k_{B} T=\ln (\rho)-1$ the free energy of an ideal gas at density $\rho$. The corresponding chemical potential is given by:

$$
\frac{\mu(\rho)}{k_{B} T}=\frac{F(\rho)}{N k_{B} T}+\frac{P(\rho)}{\rho k_{B} T} .
$$

The calculation of the chemical potential of the solid is slightly more complicated. The reason is that it is not possible to perform the integration from the ideal gas limit, as the solid melts at lower densities. One has to calculate the excess free energy of a solid at a reference density where the solid is stable, which requires a different thermodynamic integration technique, the so-called Einstein integration. The idea is to transform the solid reversibly into an Einstein crystal, where the atoms are coupled harmonically to their lattice sites. The free energy can be calculated very precisely and we use the results from Polson et al. [102] for the excess free energy of a (defect free) hard sphere solid at coexistence: $F^{e x}\left(\rho_{\text {coex }}=1.0409\right) / N k_{B} T=5.91889$. From the above equation we can then calculate the chemical potential of the solid at any other density according to:

$$
\begin{aligned}
\frac{\mu(\rho)}{k_{B} T}= & \frac{F^{i d}(\rho)}{N k_{B} T}+5.91889 \\
& +\frac{1}{k_{B} T} \int_{\rho_{\text {coex }}}^{\rho} d \rho^{\prime}\left(\frac{P\left(\rho^{\prime}\right)-\rho^{\prime} k_{B} T}{\rho^{\prime 2}}\right)+\frac{P(\rho)}{\rho k_{B} T} .
\end{aligned}
$$

For the equation of state $P(\rho)$ we used the analytical expressions by Hall [3.3] for the liquid and the solid. The integration was performed numerically.

\section{C}

\section{Surface Free Energies of Critical Nuclei}

In general, the value of the surface tension (or, more generally, surface free-energy density) depends on the criterion used to define the surface of a cluster. However, in the special case that we consider a critical nucleus, there exists a thermodynamic 
relation between the height of the nucleation barrier and the surface free-energy density associated with the thermodynamic surface of tension. Below, we derive this relation.

Consider two systems. System I contains the homogeneous, metastable phase $\beta$. System II contains the parent phase $(\beta)$ in unstable equilibrium with a critical nucleus of phase $\alpha$. We consider the general case that the parent phase is an $n$ component mixture. The height of the nucleation barrier can be computed in several ways (depending on the thermodynamic variables that we keep fixed). For instance, for a system at constant pressure and temperature, the nucleation barrier is given by the difference in Gibbs free energy between states II and I. To compute this barrier, we first evaluate the difference in the internal energy

$$
\Delta U=U^{\mathrm{II}}-U^{\mathrm{I}}
$$

The internal energy of system $\mathrm{I}$ is given by

$$
U^{\mathrm{I}}=T^{\mathrm{I}} S^{\mathrm{I}}-p^{\mathrm{I}} V^{\mathrm{I}}+\sum_{i=1}^{n} \mu_{i}^{\mathrm{I}} N_{i},
$$

where $\mu_{i}^{\mathrm{I}}$ is the chemical potential of component $i$ in state I. As state II is also in equilibrium (be it an unstable one), the chemical potentials of all species are also constant throughout the system - even though the system itself is inhomogeneous. The internal energy of system II is given by

$$
\begin{aligned}
U^{\mathrm{II}} & =T^{\mathrm{II}} S^{\mathrm{II}}-p_{\alpha}^{\mathrm{II}} V_{a}^{\mathrm{II}}-p_{\beta}^{\mathrm{II}} V_{\beta}^{\mathrm{II}}+\gamma A+\sum_{i=1}^{n} \mu_{i}^{\mathrm{II}} N_{i} \\
& =T^{\mathrm{II}} S^{\mathrm{II}}+\left(p_{\beta}^{\mathrm{II}}-p_{\alpha}^{\mathrm{II}}\right) V_{\alpha}^{\mathrm{II}}-p_{\beta}^{\mathrm{II}} V^{\mathrm{II}}+\gamma A+\sum_{i=1}^{n} \mu_{i}^{\mathrm{II}} N_{i}
\end{aligned}
$$

We consider the situation that the nucleus is formed at constant pressure and temperature. In that case, $p^{\mathrm{I}}=p_{\beta}^{\mathrm{II}}=p, T^{\mathrm{I}}=T^{\mathrm{II}}=T$ and $\mu_{i}^{\mathrm{I}}=\mu_{i}^{\mathrm{II}}=\mu_{i}$. The last equality follows because the chemical potential in the parent phase is a function of $P$ and $T$ only. The difference between the internal energies of systems I and II is then given by

$$
\Delta U=T \Delta S+\left(p-p_{\alpha}^{\mathrm{II}}\right) V_{\alpha}^{\mathrm{II}}+\gamma A-p \Delta V,
$$

where $\Delta S=S^{\mathrm{II}}-S^{\mathrm{I}}$ and $\Delta V=V^{\mathrm{II}}-V^{\mathrm{I}}$. Note that the terms involving the chemical potentials drop out of the expression for $\Delta U$. The expression for the nucleation barrier then becomes

$$
\Delta G=\Delta U+p \Delta V-T \Delta S=\left(p-p_{\alpha}^{\mathrm{II}}\right) V_{\alpha}^{\mathrm{II}}+\gamma A .
$$

This equation holds for every dividing surface. Moreover, we have not made any approximations concerning the compressibility of either phase, nor concerning the 
interfacial free energy. If we choose the surface of tension as the dividing surface, then we can use the Laplace equation $\left(\Delta p=2 \gamma_{s} / R_{s}\right.$ ) to express the height of the barrier as

$$
\Delta G=\frac{4}{3} \pi R_{s}^{2} \gamma_{s}=\frac{2 \pi}{3} \Delta p R_{s}^{3} .
$$

In what follows, it will turn out to be convenient to express the surface tension $\gamma_{s}$ in terms of the barrier height $\Delta G$ and the Laplace pressure $\Delta p$

$$
\gamma_{s}=\left(\frac{3}{16 \pi}\right)^{1 / 3} \Delta G^{1 / 3} \Delta p^{2 / 3}
$$

We stress that, for every component, the chemical potentials in the parent phase and in the critical nucleus are the same. In the absence of the Laplace pressure, the chemical potentials in phase $\alpha$ would be lower than those in phase $\beta$. The effect of the Laplace pressure is to compensate this difference for every component $i$. At first sight, it would seem that the computation of $\Delta p$ is an intractable problem for a multicomponent system - to satisfy the condition that $\mu_{i}^{\alpha}=\mu_{i}^{\beta}$ for all $i$, it is not enough to compress phase $\alpha$; we should also change its composition. The problem is greatly simplified if we make use of the semi-grand canonical ensemble. In the semigrand ensemble, the independent variables that describe the state of an $n$-component system are: the temperature $T$, the pressure $P$, the total number of particles $N$ and the set of $n-1$ differences in the chemical potential $\left(\Delta \mu_{i}\right)$ between a reference species (say, species 1 ) and all other species $i \neq 1$. The number of components $n$ can be infinite.

At coexistence, the chemical potentials of all species $i$ in the two phases, are equal: $\mu_{i}^{\alpha}=\mu_{i}^{\beta}$. In the notation of the semi-grand ensemble, this means that, at coexistence, the temperature and pressure of the two phases are equal, as are all $\Delta \mu_{i}$, and finally also the chemical potential $\mu_{1}$ of the reference compound. Now consider what happens if we supersaturate the parent phase, for instance by compression (the analysis for the case of supercooling follows by analogy). In the semi-grand ensemble we perform this supersaturation by increasing $P$, while keeping $T$ and all $\Delta \mu_{i}$ constant. Note that this route need not correspond to the physical route for supersaturation. The reason is the physical route is (usually) to supersaturate at constant composition. But in that case, all $\Delta \mu_{i}$ change by different amounts, and this is precisely the factor that complicates the analysis of nucleation in multicomponent systems.

Suppose that we have compressed the system up to a pressure $P_{\beta}$ where $\mu_{1}$ (and thereby all $\mu_{i}$ ) in the parent phase have increased by an amount $\Delta \mu^{\beta}$. An equal compression of the phase $\alpha$ leads to an increase $\Delta \mu^{\alpha}$ in the chemical potential of all species in that phase. Obviously, $\Delta \mu^{\alpha}$ is less than $\Delta \mu^{\beta}$, because beyond coexistence, phase $\beta$ is metastable. However, we can compress phase $\alpha$ to a higher pressure $P_{\alpha}$ such that

$$
\Delta \mu^{\alpha}\left(P_{\alpha}\right)=\Delta \mu^{\beta}\left(P_{\beta}\right)
$$

Note that, as we are working in the semi-grand ensemble where we keep all $\Delta \mu_{i}$ constant, we have thus achieved equality of the chemical potentials in the two phases 
for all species in the multicomponent mixture. In homogeneous nucleation, it is the Laplace pressure $\Delta p$ that ensures that the chemical potential of every individual species is equal inside and outside the critical nucleus. We can therefore make the immediate identification:

$$
\Delta p=P_{\alpha}-P_{\beta}
$$

Of course, once we have determined the pressure $P_{\Omega}$, then the density and composition of phase $\alpha$ follow.

In a simulation, we can solve Eq. 30 by making use of the fact that, for a semigrand ensemble we have the following relation:

$$
\frac{\partial \mu_{1}}{\partial P}=\frac{V}{N} .
$$

We can compute the average volume $V$ in a semi-grand simulation, and hence we can obtain $\Delta \mu$ by integration. Our expression for the Laplace pressure then becomes

$$
\int_{P_{\text {coex }}}^{P_{\beta}+\Delta P}\langle V(P)\rangle_{\alpha} d P=\int_{P_{\text {coex }}}^{P_{\beta}}\langle V(P)\rangle_{\beta} d P .
$$

This can also be written as

$$
\int_{P_{\beta}}^{P_{\beta}+\Delta P}(V(P)\rangle_{\alpha} d P=\Delta \mu^{\beta}\left(P_{\beta}\right) .
$$

For an incompressible system, we can simplify this expression further, but we will not do this here. Once we have computed $\Delta p$, we can estimate the interfacial freeenergy $\gamma_{s}$ by using our numerical information about the nucleation barrier $\Delta G$, using Eq. (29):

$$
\gamma_{s}=\left(\frac{3}{16 \pi}\right)^{1 / 3} \Delta G^{1 / 3} \Delta p^{2 / 3} .
$$

\section{References}

1. Fahrenheit DB (1724) Phil. Trans. Roy. Soc. 39:78 149

2. Tumbull D, Fischer JC (1949) J. Chem. Phys. 17:71 149, 151, 152

3. Kelton KF (1991) Solid State Physics 45:75 149, 173

4. Palberg T, (1999) J. Phys.: Condens. Matter 11:323 152, 153, 165, 166

5. Harland J, van Megen W (1997) Phys. Rev. E 55:3054 152, 153, 164, 165, 167, 187, 189,191

6. Heymann A, Stipp C, Sinn C, Palberg T (1998) J. Coll. Interf. Sci. 206:119 152,153, 165

7. Davidchack RL, Laird BB (2000) Phys. Rev. Lett. 85:4751 152, 165, 192, 195

8. Auer S, Frenkel D (2003) submitted to J. Chem. Phys. 154, 164, 172

9. Reiss H, Bowles RK (1999) J. Chem. Phys. 111:7501 154, 198 
10. ten Wolde PR (1998), Numerical Study of Pathways for Homogeneous Nucleation (available from: http://www.amolf.nl) Ph.D. thesis, University of Amsterdam, Amsterdam, The Netherlands. 154,198

11. Steinhardt PL, Nelson DR, Ronchetti M (1983) Phys. Rev. B 28:784 154

12. van Duijneveldt JS, Frenkel D (1992) J. Chem. Phys. 96:4655 154

13. ten Wolde PR, Ruiz-Montero MJ, Frenkel D (1995) Phys. Rev. Lett. 75:2714 154, 167, 184

14. Torrie GM, Valleau JP (1974) Chem. Phys. Lett. 28:578 158

15. Frenkel D, Smit B (2002) Understanding Molecular Simulations: from Algorithms to Applications, Academic Press San Diego 160, 178, 187, 201

16. Geyer CJ, Thompson EA (1995) J. Am. Stat. Assoc. 90:909 160, 164

17. Chandler D (1978) J. Chem. Phys. 68:2959 161

18. Ruiz-Montero MJ, Frenkel D,Brey JJ (1997) Mol. Phys. 90:925 161

19. Cichocki B, Hinson K (1990) Physica A 166:473 162, 163, 173

20. Medina-Noyola M (1988) Phys. Rev. Lett. 60:2705 163

21. Batchelor GK (1976) J. Fluid. Mech. 74:1 163

22. Cichocki B, Felderhof BU (1988) J. Chem. Phys. 89:1049 163

23. Beenakker CWJ, Mazur P (1983) Physica A 120:388 163

24. Tokuyama M, Oppenheim I (1994) Phys. Rev. E 50:R16 163

25. van Duijneveldt JS, Lekkerkerker HNW (1995) Science and Technology of Crystal Growth, edited van der Eerden JP and O. S. L. Bruinsma OSL, Kluwer-Academic Dordrecht 163

26. van Megen W, Underwood SM (1994) Phys. Rev. E 49:4206 163

27. Hoover WG, Ree FH (1968) J. Chem. Phys. 49:3609 164, 187, 188, 193

28. Vrij A. et al. (1983) Faraday Discuss. chem. Soc. 76:19 164

29. Pusey PN, van Megen W (1986) Nature 320:340 164, 175, 187, 188

30. Schätzel K, Ackerson BJ (1993) Phys. Rev. E 48:3766 164, 167

31. Sinn C, Heymann A, Stipp A, Palberg T (2001) Progr Coloid Polym Sci 118:266 164, $166,167,190,191$

32. Auer S, Frenkel D (2001) Nature 409:1020 164, 170, 180, 189, 190, 194, 195

33. Hall KR (1970) J. Chem. Phys. 57:2252 165, 195, 202

34. Cacciuto A, Auer S, Frenkel D (2003) accepted J. Chem. Phys. 165

35. Auer S, Frenkel D (2001) Nature 413:711 165, 170, 180

36. Cheng Z. et al. (2002) Phys. Rev. Lett. 88:015501 166

37. Dixit NM, Zukoski CF (2001) Phys. Rev. E 64:041604 166

38. Volkov I, Cieplak M, Koplik J, Banavar R (2002) Phys. Rev. E 66:061401 167

39. Cheng Z (1998) Colloidal Hard Sphere Crystallization and Glass Transition, Ph.D. thesis, Pinceton University, Princeton, US 163, 167

40. Ostwald W (1897) Z. Phys. Chem. 22:289 167

41. Alexander S, McTague JP (1978) Phys. Rev. Lett. 41:702 167

42. Klein W, Leyvraz F (1986) Phys. Rev. Lett. 57:2845 167

43. Klein W (2001) Phys. Rev. E 64:056110 167

44. Groh B, Mulder B (1999) Phys. Rev. E 59:5613 167

45. Pronk S, Frenkel D (1999) J. Chem. Phys. 110:4589 168

46. Mau SC, Huse DA (1999) Phys. Rev. E 59:4396 168

47. Pusey PN et al. (1989) Phys. Rev. Lett. 63:2753 168, 188

48. Haddon MS, Haddon SB, Poon WCK (2001) J. Phys.: Condensed Matter 13:553 168

49. Zhu J et al. (1997) Nature 387:883 168

50. O'Malley B, Snook I (2003) Phys. Rev. Lett. 90:085702 169 
51. Pusey PN (1991) Les Houches; Liquids, Freezing and Glass Transition (edited by Hansen JP, Levesque D, Zinn-Justin J), North-Holland Amsterdam 170

52. Kofke DA, Bolhuis PG (1999) Phys. Rev. E 59:618 170, 173, 188

53. Fasolo M, Sollich P (2003) Phys. Rev. Lett. 91:068301 170

54. Auer $\mathrm{S}$ (2002) Quantitative prediction of crystal nucleation rates for spherical colloids: A computational study, Ph.D. thesis, University of Amsterdam, Amsterdam, The Netherlands (available from: http://www.amolf.nl) 173

55. Viisanen Y, Strey R, Reiss H (1993) J. Chem. Phys. 99:4680 173

56. Oxtoby DW, Kashchiev D (1994) J. Chem. Phys. 100:7665 173

57. Oxtoby D (2001) Nature 413:694 173

58. Shi FG, Tong HY, Ayers JD (1995) Appl. Phys. Lett. 67:350 173, 174, 175

59. ten Wolde PR, Frenkel D (1997) Science 277:1975 173

60. Gasser U, Weeks ER, Schofield A, Pusey PN, Weitz DA (2001) Science 292:258 175, $177,182,183,184,185$

61. Auer S, Frenkel D (2002) J. Phys.:Condens. Matter 14:7667 175, 190

62. Verweij EJW, Overbeek JTHG (1948) Theory of the Stability of Lyopholic Colloids, Elsevier New York 176

63. Alexander $S$ et al. (1984) J. Chem. Phys. 80:5776 176

64. Bitzer F, Palberg T, Löwen H, Simon R, Leiderer P (1994) Phy. Rev. E 50:2821 176

65. Sirota EB, Ou-Yang HD, Sinha SK, Chaikin PM (1989) Phys. Rev. Lett. 62:1524 177

66. Robbins MO, Kremer K, Grest GS (1988) J. Chem. Phys. 88:3286 177

67. Azhar F El, Baus M, Ryckaert JP,Meijer EJ (2000) J. Chem. Phys. 112:5121 176, 177, 178,183

68. Meijer EJ, Frenkel D (1991) J. Chem. Phys. 94;2269 177

69. Dhont JKG, Smits C, Lekkerkerker HNW (1992) J. Coll. Interf. Sci. 152:386 177

70. Okubo T (1994) Macro-ion characterization: from dilute solutionto complex fluids, ACS Symposium Series 548:364 Dordrecht 177

71. Schöpe HJ, Palberg T (2002) J.Phys.Condens.Matter 14:11573 177

72. Wette P, Schöpe HJ, Liu J, Palberg T (2003) Europhys. Lett. in press 177

73. Schöpe HJ (2000) Physikalische Eigenschaften kolloidaler Festkörper Ph.D. thesis, Johannes Gutenberg Universität Mainz, Mainz, Germany 183

74. Hoogenboom JP, Derks D, Vergeer P, van Blaaderen A (2002) J. Chem. Phys. 117:11320 185

75. Auer S, Poon WCK, Frenkel D (2003) Phys. Rev. E 67:020401 186

76. de L. Costello BA, Luckham PF (1993) J.Colloid Interface Sci. 156:72 186

77. de L. Costello BA et al. (1992) Langmuir 8:464 186

78. Alexander S (1977) J. Phys. (Paris) 38:983, de Gennes PG (1988) Adv. Colloid Interface Sci. 27:189 186

79. Russel WB, Saville DA, Schowalter WR (1989) Colloidal Dispersions, Cumbridge University Press, Cambridge 187

80. Antl L et al. (1986) Colloids Surf. 17:67 187

81. Mewis J et al. (1989) A.I.Ch.E.J. 35:415, Cairns RJR et al. (1976) J. Colloid Interface Sci. 54:51 Doroszkowski A, Lambourne R (1971) J. Polym. Sci. Part C:Polym. Symp. 34:253 van Megen W, Underwood SM (1990) Langmuir 6:35 Markovic I et al. (1986) Langmuir 2:625 187, 188

82. Cebula DJ et al. (1983) Colloid Polymer Sci. 261:555 187, 188

83. Pusey PN, van Megen W (1987) Physics of Complex and Supermolecular Fluids (ed S.A. Safran and N.A. Clark, Wiley, New York) 187,188

84. Bryant $G$ et al. (2002) Phys. Rev. E 66:060501(R). 189 
85. Yethiraj A, van Blaaderen A (2003) Nature 421:513 189

86. Auer S, Frenkel D (2003) Phys. Rev. Lett. 91:015703 191

87. van Blaaderen A, Ruel R, Wiltzuis P (1997) Nature 385:321 191

88. Hoogenboom JP, van Langen-Suurling AK, Romijn J, van Blaaderen A (2003) Phys. Rev. Lett. 90:138301 191

89. Hoogenboom JP, Derks D, Vergeer P, van Blaaderen A (2002) J. Chem. Phys. 117:11320. 191

90. Hoogenboom JP, Vergeer P, van Blaaderen A (2003) J. Chem. Phys. 119:3371 191

91. Heni M, Löwen H (2000) Phys. Rev. Lett. 85:3668 191

92. Kose A, Hachisu S (1976) J. Coll. Interf. Sci. 55:487 191

93. Gast AP, Russel WB, Hall CK (1986) J. Coll. Interf. Sci. 55:161 191

94. Kaplan PD, Rouke JL, Yodh AG, Pine DJ (1994) Phys. Rev. Lett. 72:582 191

95. Dinsmore AD, Warren PB, Poon WCK, Yodh AG (1997) Europhys. Lett. 40:337 191

96. Martellozzo VC (2001) Crystallization and Phase Separationin Colloidal Systems, Ph.D. thesis, University of Edinburgh, Edinburgh, Scotland 191

97. Poon WCK, Warren PB (1994) Europhys. Lett. 28:513 191.

98. Courtemanche DJ, van Swol F (1992) Phys. Rev. Lett. 69:2078 191, 196

99. Turnbull D (1950) J. Chem. Phys. 18:198 192

100. Heni M, Löwen H (1999) Phys. Rev. E 60:7057 192

101. ten Wolde PR, Ruiz-Montero M, Frenkel D (1996) Faraday Discuss. 114:9932 198

102. Polson J.M. et al. (2000) J. Chem. Phys. 112:5339 177, 202 